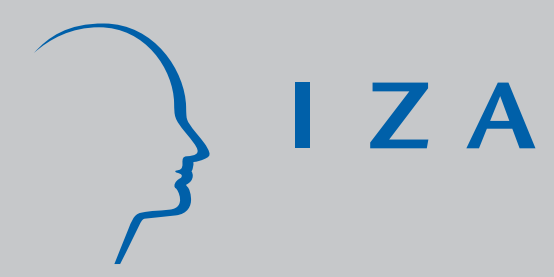

IZA DP No. 3262

Von der Frühverrentung zum längeren

Erwerbsleben: Transferleistungen, Arbeitsmarktpolitik und Weiterbildung

Werner Eichhorst

December 2007 


\title{
Von der Frühverrentung zum längeren Erwerbsleben: Transferleistungen, Arbeitsmarktpolitik und Weiterbildung
}

\author{
Werner Eichhorst \\ IZA
}

Discussion Paper No. 3262

December 2007

\author{
IZA \\ P.O. Box 7240 \\ 53072 Bonn \\ Germany \\ Phone: +49-228-3894-0 \\ Fax: +49-228-3894-180 \\ E-mail: iza@iza.org
}

\begin{abstract}
Any opinions expressed here are those of the author(s) and not those of the institute. Research disseminated by IZA may include views on policy, but the institute itself takes no institutional policy positions.

The Institute for the Study of Labor (IZA) in Bonn is a local and virtual international research center and a place of communication between science, politics and business. IZA is an independent nonprofit company supported by Deutsche Post World Net. The center is associated with the University of Bonn and offers a stimulating research environment through its research networks, research support, and visitors and doctoral programs. IZA engages in (i) original and internationally competitive research in all fields of labor economics, (ii) development of policy concepts, and (iii) dissemination of research results and concepts to the interested public.
\end{abstract}

IZA Discussion Papers often represent preliminary work and are circulated to encourage discussion. Citation of such a paper should account for its provisional character. A revised version may be available directly from the author. 
IZA Discussion Paper No. 3262

December 2007

\section{ABSTRACT}

\section{Von der Frühverrentung zum längeren Erwerbsleben: Transferleistungen, Arbeitsmarktpolitik und Weiterbildung}

Die Beschäftigung älterer Arbeitnehmer in Deutschland hat in den letzten Jahren merklich zugenommen. Die Studie untersucht diese jüngste Verbesserung der Arbeitsmarktsituation Älterer und analysiert den Beitrag, den die arbeitsmarkt- und sozialpolitischen Reformen der letzten Jahre dabei gespielt haben. Dabei steht zunächst die aktive und passive Arbeitsmarktpolitik im Mittelpunkt, bevor die Situation im Bereich der beruflichen Weiterbildung genauer untersucht wird. Diese ist zwar ein zentraler Faktor für den Erhalt der Beschäftigungsfähigkeit im Lebensverlauf, ist jedoch strukturell in Deutschland nach wie vor unterwickelt, weshalb Grundzüge einer möglichen Reform zur besseren Institutionalisierung von Anreizen für Weiterbildungsinvestitionen entwickelt werden.

JEL Classification: J68, J14

Keywords: Arbeitsmarktpolitik, Weiterbildung, Aktivierung, ältere Arbeitnehmer

Corresponding author:

Werner Eichhorst

IZA

P.O. Box 7240

D-53072 Bonn

Germany

E-mail: eichhorst@iza.org

\footnotetext{
* Der Autor dankt den Teilnehmern zweier Tagungen an der Evangelischen Akademie Loccum („Berufliche Weiterbildung Älterer in kleinen und mittleren Unternehmen“, 15.-17. Oktober 2007, und „Drei Jahre SGB II“, 10.-12. Dezember 2007) für hilfreiche Kommentare, insbesondere Christina Wübbeke.
} 


\section{Einleitung}

Die Beschäftigungssituation älterer Arbeitnehmer hat sich in Deutschland in letzter Zeit deutlich positiver als von vielen erwartet entwickelt. Dies gilt sowohl im Zeitverlauf als auch im Vergleich mit anderen europäischen Volkswirtschaften. Hierfür werden verschiedene Erklärungen struktureller und konjunktureller Art angeführt. Diese Studie legt ein Hauptaugenmerk auf den Beitrag, den die arbeitsmarkt- und sozialpolitischen Reformen der letzten Jahre zur Verbesserung der Arbeitsmarktsituation Älterer gespielt haben. Im Mittelpunkt der Reformbemühungen standen in der Vergangenheit die aktive und passive Arbeitsmarktpolitik, welche sich gegenwärtig erneut in Diskussion befinden. Daneben muss jedoch auch die Situation im Bereich der beruflichen Weiterbildung genauer untersucht werden, welche zwar ein zentraler Faktor für den Erhalt der Beschäftigungsfähigkeit im Lebensverlauf, jedoch in Deutschland wesentlich weniger stark beachtet wird.

\section{Die jüngere Entwicklung bei der Beschäftigung Älterer}

Bereits seit einigen Jahren ist die Frage der Verlängerung des Erwerbslebens einer der zentralen Streitpunkte der Arbeitsmarkt- und Sozialpolitik. Mit der Entscheidung, die Regelaltersgrenze für jüngere Jahrgänge auf 67 Jahre anzuheben, wurde ein weiterer Schritt in diese Richtung vollzogen. Damit soll auf der einen Seite das Rentensystem durch längere Einzahlungen und kürzere Auszahlungsphasen stabilisiert werden, auf der anderen Seite das Arbeitskräftepotenzial älterer Erwerbspersonen produktiver genutzt werden. Beides soll die erwarteten Effekte des de-

mographischen Wandels lindern helfen. Jedoch sind gleichzeitig auch Bestrebun- 
gen verstärkt worden, die Verlängerung des Erwerbslebens durch neue Formen eines flexiblen Renteneintritts und verschiedene Optionen zum vorzeitigen Ausstieg aus dem Arbeitsmarkt zu flankieren und faktisch aufzuweichen. Einerseits könnte die Verlängerung des Erwerbslebens, so wird häufig argumentiert, zu Lasten der älteren Arbeitnehmer gehen, die aufgrund von gesundheitlichen oder qualifikatorischen Defiziten nicht so lange auf dem Arbeitsmarkt mithalten können. Andererseits wird auf die mögliche Verdrängung von jüngeren Arbeitskräften hingewiesen. Gerade dieses Argument war in der deutschen Diskussion über die Beschäftigung Älterer in den letzten zehn bis zwanzig Jahren eine der zentralen Begründungen für verschiedene Modelle des vorgezogenen Ruhestands oder eines gleitenden Übergangs in die Altersrente, z.B. durch Altersteilzeit, um für jüngere Arbeitskräfte „Platz zu machen“.

Die Arbeitsmarktsituation für Ältere ist mittlerweile jedoch längst nicht mehr so schlecht, wie häufig vermutet wird. Dies zeigt sich im Hinblick auf drei zentrale Indikatoren: a) beim Niveau und bei der Struktur der Beschäftigung, b) beim Zeitpunkt des faktischen Ausscheidens aus dem Arbeitsmarkt und c) bei der Entwicklung der Arbeitslosigkeit Älterer.

In Deutschland wurden in den letzten Jahren im Bereich der Beschäftigung Älterer enorme Fortschritte erzielt. Abbildung 1 zeigt die Beschäftigungsquoten der 55 bis 64-Jährigen für eine Auswahl europäischer Staaten (vgl. hierzu auch European Commission 2007). Deutschland ist 2006 bei der Beschäftigungsquote Älterer bereits fast auf 49 Prozent gekommen. Im zweiten Quartal 2007 ist mit einer Beschäftigungsquote der 55- bis 64-Jährigen von 52,0\% die 50-Prozent-Marke, die Zielvorgabe der Europäischen Beschäftigungsstrategie für das Jahr 2010, erstmals überschritten worden (Bundesagentur für Arbeit 2007). Das ist ein durchaus beachtlicher Fortschritt im Vergleich zur Jahrtausendwende und zur Situation in den spä- 
ten 1990er Jahren, als weniger als 40\% der Älteren erwerbstätig waren. Im Zeitvergleich von 2000 und 2006 hat Deutschland den höchsten Zuwachs bei der Beschäftigung älterer Arbeitskräfte im Vergleich mit anderen dargestellten Ländern zu verzeichnen. Spitzenreiter sind bezogen auf das zweite Quartal 2007 jedoch nach wie vor Schweden (69,9\%), Dänemark (58,7\%) und Großbritannien (57,4\%).

\section{ABBILDUNG 1 HIER}

Die Beschäftigungssituation der älteren Arbeitskräfte ist in Deutschland zurzeit als sehr dynamisch zu bewerten. Im internationalen Vergleich fällt der Anstieg stärker aus als in einer Reihe anderer Staaten, die üblicherweise als Vorreiter bei der verstärkten Beschäftigung Älterer angesehen werden. Weiterhin ist aber zu beobachten, dass es nach wie vor ein Defizit der Beschäftigung Älterer im Verhältnis zur Beschäftigungsquote aller im erwerbsfähigen Alter, d.h. Personen zwischen 15 und 64 Jahren, gibt. Allerdings geht diese Differenz in allen Ländern zurück. Die Beschäftigungsquote der Älteren nähert sich so immer stärker der durchschnittlichen Beschäftigungsquote an. Auch hier zeigt sich deutlich, dass Deutschland zu den Ländern zählt, in denen dieser Trend am stärksten ausgeprägt ist. Von 2000 bis 2006 konnte der Rückstand der Älteren um neun Prozentpunkte verringert werden. Das ist ein beachtlicher Fortschritt, wenngleich Deutschland bei der Differenz des Beschäftigungsniveaus immer noch deutlich schlechter abschneidet als beispielsweise Norwegen oder Schweden.

\section{ABBILDUNG 2 HIER}


Gleichzeitig hat die sozialversicherungspflichtige Beschäftigung der Älteren deutlich zugenommen. Früher war der Beschäftigungszuwachs bei den Älteren in Deutschland sehr stark von geringfügiger Beschäftigung getrieben (Büttner 2005). Dieses Muster hat sich - wie auch am Arbeitsmarkt insgesamt - inzwischen verändert. In Abbildung 3 wird ein Indexwert ausgewiesen, bei dem das Jahr 2000 dem Ausgangsniveau von 100 entspricht. Für die gesamte Gruppe der 50- bis 64Jährigen zeigt sich eine recht robuste Aufwärtsbewegung, die bis 2006 zu einem Zuwachs um etwa ein Sechstel gegenüber der Situation im Jahr 2000 geführt hat. Im Bereich der 55- bis 60-Jährigen, die teilweise den Arbeitsmarkt über noch bestehende Ausstiegsoptionen, z.B. das längere Arbeitslosengeld I, verlassen haben, findet sich zwar ein leichter Rückgang, dem steht aber die relativ deutliche Aufwärtsbewegung gerade bei den älteren Jahrgängen innerhalb dieser Gruppe gegenüber. Der Beschäftigungszugewinn bei den Älteren trägt gegenwärtig mit etwa zwei Dritteln sehr deutlich zum Wachstum der Beschäftigung in Deutschland insgesamt bei (Bundesagentur für Arbeit 2007).

\section{ABBILDUNG 3 HIER}

In Tabelle 1 ist die Qualifikationsstruktur der älteren Arbeitskräfte dargestellt. Dies belegt, dass sich diese Struktur von 1996 bis 2004 ein Stück weit verschoben hat. Die Anzahl der höher Qualifizierten in den rentennahen Jahrgängen hat, im Gegensatz zu früher, zugenommen, was auch zum Beschäftigungsaufbau, den wir bei den Älteren insgesamt beobachten können, beiträgt. Höher qualifizierte Personen mit einer soliden Erwerbsbiographie sind mit größerer Wahrscheinlichkeit auch 
länger im Arbeitsmarkt präsent (Brenke 2007). Aber auch bei den älteren Erwerbstätigen mit einem geringeren Bildungsstand ist ein Zuwachs der Beschäftigung zu beobachten.

TABELLE 1 HIER

Auch beim Erwerbsaustrittsalter gab es zwischen 2001 und 2006 einen merklichen Anstieg zu verzeichnen, wie Abbildung 4 darlegt. Im Jahr 2006 verließen Arbeitnehmer in Deutschland im Durchschnitt mit 61,9 Jahren den Arbeitmarkt, noch 2001 war dies bereits mit 60,6 Jahren der Fall gewesen. Somit nähert sich das faktische Erwerbsaustrittsalter langsam dem jetzt noch geltenden gesetzlichen Rentenalter von 65 Jahren an. Dieser Trend ist nicht in allen Ländern in gleichem Maße ausgeprägt, teilweise sind in Ländern mit einem hohen Beschäftigungsanteil Älterer Stagnation oder Rückschritte zu verzeichnen, aber Deutschland bewegt sich auch hier auf einem stabilen Pfad der Verbesserung.

ABBILDUNG 4 HIER

Gleichwohl gilt noch immer, dass Personen, die in einem späten Abschnitt des Erwerbslebens arbeitslos werden, im Vergleich zu Jüngeren größere Gefahr laufen, längere Zeit arbeitslos zu sein. Dies ist nicht nur in Deutschland zu beobachten, sondern es handelt sich hierbei um einen Befund, der sich in einer Reihe anderer Industriestaaten ebenso deutlich niederschlägt. So sank zwar die Arbeitslosigkeit Älterer in Deutschland parallel zum Beschäftigungszuwachs relativ konsistent ü- 
ber die letzten zwei Jahre hinweg. 2006 ging die Arbeitslosigkeit im SGB III bei den über 50 jährigen Erwerbspersonen um zehn Prozent und im Jahr 2007 bis November um weitere 22,8\% zurück; im SGB II, also bei den Langzeitarbeitslosen, gab es nach einem Zuwachs um 2,1\% noch im Jahr 2006 bis zum November 2007 lediglich einen Rückgang der Arbeitslosigkeit um 5,4\%. In Abbildung 5 sieht man den Anteil der Langzeitarbeitslosen, die länger als zwölf Monate arbeitslos sind, an allen Arbeitslosen über 50 Jahren im Vergleich zum Anteil der Langzeitarbeitslosen bei den unter 50-Jährigen. In den meisten Ländern schneiden die älteren Arbeitskräfte deutlich schlechter ab. Nach wie vor besteht ein Problem bei der Arbeitsmarktintegration der Älteren, das sich in einer im Durchschnitt längeren Arbeitslosigkeit und einer weniger großen Wahrscheinlichkeit ausdrückt, als älterer Arbeitsuchender noch einmal einen anderen Arbeitsplatz zu finden. Dies gilt vor allem im Bereich der Grundsicherung (SGB II), weniger im Bereich der Arbeitslosenversicherung (SGB III). Ältere Arbeitslose über 50 Jahren sind im Durchschnitt 66,9 Wochen arbeitslos, alle Arbeitslosen im Mittel nur 41,9 Wochen.

\section{ABBILDUNG 5 HIER}

Der Rückgang der Arbeitslosigkeit älterer Erwerbspersonen im SGB III ist eher mit einem geringeren Zustrom als mit einem verstärkten Abstrom aus der Arbeitslosigkeit zu erklären. Dies kann dahin gehend interpretiert werden, dass in erster Linie die Weiterbeschäftigung Älterer zunimmt, was sich etwa im starken Wachstum der unbefristeten Vollzeitbeschäftigung von Älteren niederschlägt. Im SGB II haben die Abgänge aus der Arbeitslosigkeit zwar stärker zugenommen, jedoch sind Übergänge in Beschäftigung etwas seltener geworden. Dies bedeutet, dass sich die 
Dauer des Erwerbslebens für viele Beschäftigte verlängert, es aber nach wie vor Wiedereinstiegsprobleme im höheren Lebensalter nach einem einmal eingetretenen Arbeitsplatzverlust gibt. Darin spiegelt sich jedoch auch die längere Bezugsdauer des Arbeitslosengeldes I wider, da längere Anspruchsdauern generell mit einem längeren Verbleib in der Arbeitslosigkeit einhergehen und die Suchintensität vermindern. Eintritte in Beschäftigung fallen bei den älteren Arbeitskräften noch immer geringer aus als bei den jüngeren.

Wichtig im Zusammenhang mit der Diskussion über die Frühverrentung ist die Tatsache, dass der Austritt aus dem Arbeitsmarkt und der Übergang in den vorgezogenen Ruhestand und damit die Inaktivität meist irreversibel sind, d.h. dass dann nur noch eine relativ geringe Wahrscheinlichkeit besteht, noch einmal in den Arbeitsmarkt zurückzukehren. Das ist noch problematischer als Arbeitslosigkeit selbst, da während dieser im Prinzip noch eine Verfügbarkeit für den Arbeitsmarkt samt Zugang zu Arbeitsvermittlung und Fördermaßnahmen besteht, während der Übergang in den Vorruhestand, also Inaktivität, in der Regel ein dauerhaftes Ausscheiden aus dem Arbeitsmarkt bedeutet (OECD 2006).

\section{ABBILDUNG 6 HIER}

Aus den vorliegenden Daten ist zu schließen, dass die Älteren derzeit sehr stark von der Verbesserung der Arbeitsmarktlage in Deutschland profitieren. Beim Beschäftigungszuwachs ist Deutschland innerhalb Europas sogar Spitzenreiter. Außerdem erweisen sich in der öffentlichen Diskussion über lange Zeit etablierte Argumentationsmuster, dass der Arbeitsmarkt für Ältere faktisch verschlossen sei und Ältere keinen Job mehr finden könnten und den Arbeitsmarkt möglichst 
schnell verlassen sollten, jetzt mehr noch als in der Vergangenheit als widerlegbar. Auch das Argument, ältere Arbeitskräfte nähmen den Jüngeren Arbeit, erweist sich als irreführend, wie Abbildung 7 zeigt. Vielmehr kann man derzeit beobachten, dass bestimmte Frühverrentungsroutinen, die über die letzten Jahrzehnte etabliert wurden, derzeit erodieren und einem konstruktiveren Zugang zur Beschäftigung Älterer weichen.

ABBILDUNG 7 HIER

\section{Kompensation: Aktive Arbeitsmarktpolitik und Transferleistungen}

Es gibt eine Reihe struktureller Faktoren, die diesen positiven Trend bei der Beschäftigung Älterer vorantreiben. Man kann die Verbesserung der Beschäftigungssituation Älterer über mehrere Jahre hinweg nicht allein durch die momentane konjunkturelle Gunst erklären. Ebenso wenig gilt dies für langfristige Unterschiede im Beschäftigungsniveau älterer Erwerbspersonen im internationalen Vergleich. Die Veränderungen im Bereich der Transferleistungen müssen dabei ebenso in Betracht gezogen werden wie die Rolle der aktiven Arbeitsmarktpolitik (Hirschenauer 2007). Daneben stellt sich das Problem der beruflich relevanten Aus- und Weiterbildung als zentrale Schlüsselfrage, genauso wie die Frage nach Arbeitsschutz, Arbeitsplatzgestaltung, Gesundheit am Arbeitsplatz, Lohn- und Tarifpolitik sowie der Senioritätsentlohnung (vgl. hierzu auch Eichhorst 2006). 


\subsection{Transferleistungen für Ältere}

Die Anreize, vorzeitig aus dem Arbeitsmarkt auszuscheiden, sind in Deutschland über die letzte Dekade hinweg Stück für Stück beschnitten worden. Das hat mittelbar den Weg für die jetzt günstigere Situation älterer Arbeitskräfte auf dem Arbeitsmarkt bereitet. Das gilt sowohl für die Beschneidung der verschiedenen Optionen zum vorzeitigen Rentenbezug im Bereich der Altersrente und der Renten bei Erwerbsunfähigkeit bzw. Erwerbsminderung als auch für die Verkürzung des Arbeitslosengeldes I von 32 auf 18 Monate bei den über 55-Jährigen und mittelbar auch für die Abschaffung der einkommensbezogenen Arbeitslosenhilfe Ende 2004. Damit verhalten sich die Effekte der Kürzung der Bezugsdauer spiegelbildlich zu deren Verlängerung in den achtziger Jahren (Steiner 1997).

Wird der vorzeitige Ausstieg aus dem Arbeitsmarkt weniger stark öffentlich gefördert, so ändert sich das Verhalten von Arbeitnehmern und Arbeitgebern in dem Sinne, dass Wieder- und Weiterbeschäftigung auch jenseits der 55 Jahre wieder häufiger werden. Auch der mittelfristige Übergang zur Rente mit 67 und die generelle Senkung der Leistungen aus der gesetzlichen Rentenversicherung für jüngere Kohorten wirken in diese Richtung. Weniger attraktive Alternativen im Transfersystem führen zu einem Rückgang der Anspruchslöhne und einer höheren Suchintensität bei Arbeitslosigkeit. Gleichzeitig legt der Kündigungsschutz, den ältere Beschäftigte innehaben, eine Weiterbeschäftigung nahe, sofern die Arbeitgeber nicht bereit sind, im Falle einer Auflösung des Beschäftigungsverhältnisses erhebliche Zusatzleistungen, d.h. Abfindungen, zu den weniger großzügigen Transferzahlungen zu bieten. Diese beiden Faktoren haben sich gegenüber der Situation Anfang der Dekade grundsätzlich verändert. 
Allerdings gibt es noch immer mindestens zwei Elemente, die nicht konsistent in dieses Muster passen: die besonderen Regelungen zum Bezug von Arbeitslosenunterstützung und die öffentlich geförderte Altersteilzeit. Beide berechtigen zu einem vorgezogenen Wechsel in die Altersrente (nach Altersteilzeitarbeit oder wegen Arbeitslosigkeit), wobei der frühestmögliche Zeitpunkt zum Renteneintritt mit Abschlägen derzeit noch bis Ende 2008 auf 63 Jahre angehoben wird. Beide Rentenarten sind bereits derzeit ohne Abschläge jedoch erst ab 65 Jahren verfügbar (Hirschenauer 2007).

Zum einen ist die Regelung nach $\S 428$ SGB III Ende 2005 noch einmal für zwei Jahre verlängert worden und läuft Ende 2007 erneut aus, wobei eine weitere Verlängerung, evtl. mit Modifikationen, zu erwarten ist. Das heißt, dass ältere Arbeitslose Arbeitslosengeld I und Arbeitslosengeld II auch dann beziehen können, ohne dem Arbeitsmarkt zur Verfügung zu stehen, wenn sie zum nächstmöglichen Zeitpunkt einen Rentenantrag stellen und eine Rente ohne Abschlag in Anspruch nehmen. Dies umfasst derzeit etwas mehr als 200.000 Personen und stellt eine der größten Personengruppen im Übergang von der Erwerbstätigkeit in den Ruhestand dar (Brussig/Wojtkowski 2007). Analog gilt dies nach § 65 Abs. 4 auch im SGB II für Bezieher des Arbeitslosengeldes II und betrifft ebenfalls rund 200.000 grundsätzlich noch erwerbsfähige Personen (Bundesagentur für Arbeit 2007). Allerdings hätte bei einem Wegfall dieser Regelung nach dem Prinzip der Nachrangigkeit der Grundsicherung gegenüber Rentenansprüchen ein frühzeitiger Wechsel in das vorrangige Rentensystem erfolgen müssen, notfalls auch unter Inkaufnahme von Rentenabschlägen. Die Optionen von § 428 SGB III und § 65 Abs. 4 SGB II treffen auf entsprechende Motivationslagen der Leistungsbezieher, die entweder für sich keine Chancen mehr auf dem Arbeitsmarkt sehen oder sich dem „Fordern und Fördern“ nicht mehr aussetzen wollen, bei welchem aber die Älteren ohnehin 
vernachlässigt werden. Die Möglichkeit eines erleichterten Leistungsbezugs von Arbeitslosengeld I oder II im Vorfeld des Rentenbezugs steht einer effektiveren Fördern und Fordern auch der Älteren im Wege, die im Grunde zu einem erheblichen Teil noch erwerbsfähig und aktivierbar wären.

Die Sonderregelungen fallen Anfang 2008 jedoch wohl nicht ersatzlos weg. Die Nachfolgeregelung, die derzeit vorbereitet wird, bedeutet für Neueintritte, dass Arbeitsuchende, denen nicht innerhalb von 12 Monaten ein Arbeitsangebot gemacht werden kann, zwar weiterhin bis zum Renteneintritt nicht mehr als arbeitsuchend gelten werden. Ihnen stehen aber auf eigenen Wunsch sämtliche Integrationsangebote der Arbeitsagenturen und der Träger des SGB II zur Verfügung. Es soll regelmäßig geprüft werden, ob Förder- oder Arbeitsangebote gemacht werden können. Zweifelhaft bleibt dabei aber, wie "aktivierend“ dies in der Praxis gehandhabt wird. Ein Übergang in eine vorzeitige Rente mit Abschlägen statt Arbeitslosengeld II kommt vor dem 63. Lebensjahr jedoch nicht in Betracht. Eine Rente ab 63 ist nur möglich, wenn die rentenrechtlichen Voraussetzungen erfüllt sind. Frauen und Menschen mit einer Schwerbehinderung können bisher noch unter bestimmten Voraussetzungen vor dem 63. Lebensjahr und damit früher als andere Versicherte in Rente gehen. Liegt eine besondere Härte vor, dann ist der Verweis auf einen vorgezogenen Rentenbezug nicht zulässig. Dies gilt beispielsweise für Menschen, die als so genannte „Aufstocker" zu ihrem Arbeitseinkommen ergänzend Arbeitslosengeld II beziehen.

Zum anderen gibt es nach wie vor für Beschäftigte ab 55 Jahren die öffentlich geförderte Altersteilzeit, welche zu mehr als $85 \%$ als so genanntes „Blockmodell“ mit einer Phase der Vollzeittätigkeit und einer Phase der Freistellung, d.h. Nichtarbeit, genutzt wird, so dass in der Praxis nicht von einem gleitenden Übergang in den Ruhestand gesprochen werden kann. Auch die Altersteilzeit ist ein Instrument, das 
im Prinzip befristet ist, sich aber als relativ zählebig erwiesen hat. Derzeit sind noch bis Ende 2009 Neueintritte in die geförderte Altersteilzeit möglich, so dass eine weitere Kohorte älterer Arbeitnehmer in Altersteilzeit eintreten kann. Auch hier ist eine weitere Verlängerung in der Diskussion.

Das vorzeitige Ausscheiden aus dem Arbeitsmarkt ist jedoch insgesamt deutlich weniger attraktiv geworden. Der Ausstieg wird weniger stark mit Mitteln der Sozialversicherung subventioniert als in der Vergangenheit. Für Erwerbstätige ist es unattraktiver geworden, den Arbeitsmarkt relativ früh zu verlassen, sofern der Arbeitgeber nicht zusätzliche Ressourcen in eine solche Anpassungsstrategie investiert. Die Statussicherung durch Lohnersatzleistungen ist bei längerer Arbeitslosigkeit auch für ältere Arbeitskräfte nur noch sehr eingeschränkt möglich. Wie stark ausgeprägt dieser Gedanke aber ist, sieht man an der jüngsten Auseinandersetzung um die Verlängerung des Arbeitslosengeldes I und am Beschluss des Parlaments vom November 2007, ab 2008 eine erneute Verlängerung des Bezugsdauer für ältere Arbeitslose von 18 auf 24 Monate vorzunehmen. Über 50-Jährige erhalten künftig 15 Monate Arbeitslosengeld I, ab 55 Jahren beträgt die Bezugsdauer 18 Monate, ab 58 ganze zwei Jahre. Hier geht es gerade darum, das statussichernde Element wieder zu stärken und ein Stück weit die Situation wieder herzustellen, wie sie bis Anfang 2006 gegeben war.

Nach dem künftigen Recht können also ältere Arbeitslose über 58 zunächst zwei Jahre die Versicherungsleistung Arbeitslosengeld I beziehen und danach Arbeitslosengeld II, wobei zwei Jahre lang noch ein befristeter und degressiv gestalteter Zuschlag gewährt wird. Über 58 Jahre alte Bezieher von Arbeitslosengeld II müssen dem Arbeitsmarkt dabei im Prinzip nicht mehr zur Verfügung stehen. Soll eine Phase in der bedürftigkeitsabhängigen Grundsicherung ganz vermieden werden, so ist ein Wechsel in das wieder verlängerte Arbeitslosengeld I jedoch erst zu ei- 
nem späteren Zeitpunkt attraktiv, nämlich zwei Jahre vor dem Übergang in eine abschlagsfreie Rente, also ab 63 Jahren. Grundsätzlich ist aber damit zu rechnen, dass ältere Arbeitslose angesichts dieser Brückenlösung in den Transferleistungen in Zukunft noch immer weniger stark aktiviert werden als jüngere und sich auch künftig vor dem gesetzlichen Renteneintrittsalter aus dem Arbeitsmarkt verabschieden.

\subsection{Der Beitrag der aktiven Arbeitsmarktpolitik}

Im Bereich der aktiven Arbeitsmarktpolitik besteht eine erstaunliche Vielfalt an Instrumenten, die sich speziell um die Förderung der Beschäftigung Älterer kümmern sollen. Dabei lassen sich drei Typen unterscheiden: a) die Förderung der Beschäftigungsaufnahme durch Subventionen für Arbeitgeber und Arbeitnehmer, b) die Förderung der beruflichen Weiterbildung und c) öffentliche Beschäftigungsangebote.

Unter die Maßnahmen zur Förderung der Beschäftigungsaufnahme speziell für Ältere fallen besonders großzügige Eingliederungszuschüsse an Arbeitgeber nach $\S 421 \mathrm{f}$ SGB III, der Beitragsbonus in der Arbeitslosenversicherung für Arbeitgeber, die einen älteren Arbeitslosen einstellen (§ 421 k SGB III) und die Entgeltsicherung für Arbeitnehmer nach $\S 421$ j SGB III, d.h. eine Aufstockung geringer Löhne, wenn man einen niedriger entlohnten Arbeitsplatz annimmt. Maßnahmen wie der Beitragsbonus oder die Eingliederungszuschüsse stellen eine Bezuschussung der Arbeitskosten auf der Seite des Arbeitgebers dar, während die Entgeltsicherung niedrigere Löhne für ältere Arbeitsuchende attraktiver machen soll. Entgeltsicherung und Beitragsbonus sind mit den Hartz-Reformen eingeführt worden und 
wurden zum Teil im Zuge der „Initiative 50 Plus“ erweitert und verlängert, z.B. wurde die Entgeltsicherung etwas großzügiger ausgestaltet.

Daneben besteht nach $\S 417$ Abs. 1 SGB III eine gezielte Maßnahme zur Förderung der Weiterbildung von älteren Arbeitskräften in kleinen und mittelgroßen Betrieben, soweit diese Bildungsmaßnahmen außerhalb des Betriebes stattfinden und es sich um allgemeine Qualifikationen handelt. Auch diese Maßnahme wurde zuletzt verlängert und ausgedehnt, so dass jetzt auch etwas größere Betriebe mit bis zu 250 Beschäftigten (früher: 100) auf die Förderung der Weiterbildung von älteren Beschäftigten ab 45 Jahren (früher: 50 Jahren) zurückgreifen können. Hinzu kommen weitere Instrumente, die zur Förderung der Weiterbildung Älterer genutzt werden können, etwa die Weiterbildung Ungelernter nach § 235c SGB III oder die Jobrotation (§ $229 \mathrm{ff}$ SGB III). Auch hat die Bundesagentur mit der Initiative „WeGebAU“ Maßnahmen zur Weiterbildung von Älteren und Geringqualifizierten 2006 und 2007 gebündelt und gezielt unterstützt (Lott/Spitznagel 2007).

Parallel hierzu wurden 30.000 Zusatzjobs für Ältere geschaffen, um über öffentlich finanzierte Beschäftigung die Arbeitslosigkeit Älterer gering zu halten. Darüber hinaus werden im SGB II vor allem die so genannten „Ein-Euro-Jobs“ als Instrument auch für Ältere eingesetzt. Für schwer vermittelbare Arbeitslose im Arbeitslosengeld II, nicht nur ältere, wurden darüber hinaus zum Oktober 2007 neu artige „Beschäftigungszuschüsse“ nach § 16a SGB II eingeführt. Langzeitarbeitslose, die trotz mindestens sechsmonatiger Aktivierung nicht erfolgreich in Beschäftigung gebracht werden können und von denen dies in absehbarer Zeit auch nicht mehr erwartet werden kann, können mit Eingliederungszuschüssen bis zu 75\% eines tariflichen oder ortsüblichen Entgelts für zwei Jahre gefördert werden, was ggf. auch unbegrenzt verlängert werden kann. 
Schließlich gibt es mittlerweile eine Neufassung der Befristungsregelung, d.h. der Möglichkeit, ältere Arbeitlose ohne sachlichen Grund befristet einzustellen und somit potenzielle Einstellungshemmnisse zu umgehen. Diese war notwendig geworden, nachdem der Europäische Gerichtshof die zwischenzeitlich geltende Bestimmung verworfen hatte. Der EuGH hatte argumentiert, dass die bisherige Regelung mittelbar eine Diskriminierung Älterer sei, weil sie durch die Möglichkeit zur sachgrundlosen Befristung faktisch keinen Zugang mehr zum Kündigungsschutz hätten. Seit Mai dieses Jahres gibt es eine Neuregelung, welche besagt, dass bei Erwerbslosen nach Maßnahmenteilnahme eine sachgrundlose Befristung von älteren Arbeitskräften wieder möglich sein soll.

Die aktive Arbeitsmarktpolitik leistet zur Beschäftigungsförderung Älterer insgesamt jedoch nur einen marginalen Beitrag. Dies zeigt sich bereits bei den Teilnehmerzahlen. Die Inanspruchnahme gerade der konzeptionell innovativeren Instrumente fällt sehr gering aus. Es gibt beispielsweise gegenwärtig etwa 5.000 Fälle in der Entgeltsicherung, 2.000 in der beruflichen Weiterbildung von älteren Beschäftigten und 7.000 in der Weiterbildung von älteren Arbeitslosen. Dem standen über 400.000 im Bereich des § 428 SGB III bzw. § 65 Abs. 4 SGB II sowie 100.000 Personen in Altersteilzeit sowie 77.000 Ältere in Arbeitsgelegenheiten gegenüber. Im Programm „WeGebAU“ waren im Jahr 2006 lediglich rund 2.000 Zugänge von Beschäftigten (neben 110.000 Arbeitslosen) zu verzeichnen (Lott/Spitznagel 2007).

Die speziellen Regelungen in den Transferleistungen spielen also insgesamt eine wesentlich größere Rolle als die aktiven Fördermaßnahmen. Gleichzeitig sind Ältere in Maßnahmen der öffentlich geförderten Beschäftigung mit geringen Eingliederungschancen stärker vertreten als in Bildungsmaßnahmen, präventiv angelegten Maßnahmen und den mit Hartz neu eingeführten Fördermöglichkeiten. Die Möglichkeit, in den Bezug von Transferleistungen ohne Verfügbarkeit für den Ar- 
beitsmarkt wechseln zu können, steht einer stärkeren Teilnahme an Bildungsmaßnahmen also eher entgegen.

Weiterhin belegen die Evaluationsstudien der Hartz-Reformen, dass die Maßnahmen im Bereich der aktiven Arbeitsmarktpolitik für Ältere keine nennenswerten Effekte gehabt haben. Einige Maßnahmen wie Beitragsbonus und Entgeltsicherung wurden kaum genutzt und zeitigten keine signifikanten Wirkungen. Die Eingliederungszuschüsse werden zwar stärker nachgefragt und erzeugen auch stärkere Eingliederungseffekte, sind aber stark für Mitnahme- und Substitutionseffekte anfällig (ZEW/IAB/IAT 2006, Ammermüller et al. 2006). Vorliegende Studien beziehen sich zwar zunächst nur auf die Instrumente im Bereich des SGB III, doch deuten vorläufige Erkenntnisse auf ähnliche Effekte bei Zielgruppen des SGB II hin.

Die Wirkungen der aktiven Maßnahmen sind also durchaus ambivalent einzuschätzen, so dass von ihnen auch künftig keine nennenswerten Effekte auf die Verbesserung der Arbeitsmarktchancen Älterer zu erwarten sind, auch nicht durch eine Ausweitung von arbeitsmarktpolitischen Maßnahmen für Ältere oder die Einführung neuer Instrumente. Darüber hinaus stellt sich aktive Arbeitsmarktpolitik speziell für Ältere als grundsätzlich problematisch dar, weil sie ihnen pauschal bestimmte Defizite nahe legt. In diesem Sinne kann sie der verbreiteten „Defizittheorie“ des Alters und der Wahrnehmung älterer Arbeitnehmer als "Problemgruppe“ mittelbar sogar Vorschub leisten. Gerade die Gewährung von Eingliederungszuschüssen trägt dazu bei, dass Arbeitgeber häufig nur noch geförderte Einstellungen von Älteren vornehmen und die Subventionierung gleichsam als selbstverständlich ansehen. Subventionierte Beschäftigung und Bildungsmaßnahmen für Ältere setzen dabei grundsätzlich zu spät an und versuchen, einmal eingetretene Qualifikationsdefizite auszugleichen und Arbeitslosigkeit zu kompensieren. 
Der derzeit diskutierte öffentlich finanzierte oder „soziale“ Arbeitsmarkt, in den auch Zusatzjobs für Ältere, „Ein-Euro-Jobs“ und der neue „Beschäftigungszuschuss“ fallen, welche überproportional stark auf Ältere angewandt werden, ist keine Brücke zurück in den ersten, sondern kann allenfalls als „Marktersatz“ interpretiert werden. Aus verschiedenen Evaluationsstudien zu ähnlich gestalteten Beschäftigungsinstrumenten ist bekannt, dass hierdurch nur mit geringer Wahrscheinlichkeit auf Aufnahme einer sozialversicherungspflichtigen Beschäftigung am regulären Arbeitsmarkt gerechnet werden kann. Insgesamt sind die Veränderungen im System der Transferleistungen wesentlich bedeutender als der Beitrag der aktiven Arbeitsmarktpolitik zur derzeit zu beobachtenden Verbesserung der Beschäftigungssituation älterer Arbeitskräfte.

\section{Prävention: Weiterbildung und „lebenslanges Lernen“}

Im Folgenden soll auf einige Daten zur Weiterbildungssituation in Deutschland, wie man sie aus international vergleichenden und standardisierten Befragungen erkennen kann, eingegangen werden. Zunächst gibt es Zahlen von Eurostat über einen längeren Zeitraum hinweg bis zum Jahre 2005. Sie zeigen, dass die Weiterbildungsbeteiligung insgesamt in Deutschland im Vergleich zu anderen Staaten relativ gering ausfällt. Man kann hier von etwa fünf bis sieben Prozent der Erwerbstätigen ausgehen, die angeben, im Referenzzeitraum von vier Wochen vor der Befragung an einer Weiterbildung teilgenommen zu haben.

Weiterhin gibt es Angaben zur Intensität der Beteiligung an Weiterbildung. Hier zeigen sich für Deutschland im Grunde zwei Merkmale. Zum einen sinkt die Beteiligung an Weiterbildung mit höherem Lebensalter stark ab. Das ist mit Blick auf 
die Struktur, die wir bis vor kurzem innerhalb des Arbeitsmarktes und der institutionellen Rahmenbedingungen für die Beschäftigung Älterer hatten, nicht erstaunlich. Weiterbildung ist für Arbeitgeber und Arbeitnehmer umso lohnender, je länger mit einem Verbleib im Arbeitsmarkt zu rechnen ist. Zum anderen können wir feststellen, dass die Häufigkeit und Intensität von Weiterbildung sehr stark vom formalen Bildungsniveau der Erwerbstätigen abhängt. Je höher die Qualifikation, umso stärker die Beteiligung an Weiterbildung. Bei Anwendung der Dreigliederung der OECD nach niedrigem (ohne weiterführenden Schul- oder Berufsabschluss), mittlerem (mit Berufsabschluss oder Abitur) und hohem Bildungsniveau (akademischer Abschluss) ergibt sich, dass sich Weiterbildung in Deutschland sehr stark auf die Höchstqualifizierten konzentriert. In dieser Gruppe sind die Teilnehmerzahlen relativ stabil über den Lebensverlauf hinweg, während es bei den Geringerqualifizierten zu einem sehr starken Absinken im Verlauf des Erwerbslebens kommt.

\section{TABELLEN 2, 3 UND 4 HIER}

Interessant ist in diesem Zusammenhang eine einfache Gegenüberstellung der Teilnahme an Weiterbildung mit der noch zu erwartenden Verweildauer im Arbeitsmarkt, hier gemessen als tatsächliches Alter des Ausscheidens aus dem Arbeitsmarkt (Abbildung 8). Es gibt einen positiven Zusammenhang dahingehend, dass Personen, die eine Perspektive auf eine noch lange Verweildauer im Arbeitsmarkt haben, doch in wesentlich stärkerem Maße als andere Gruppen an Weiterbildung partizipieren werden. Dies ist letztlich ein Zusammenhang, der sich wechselseitig stabilisiert: Weiterbildung trägt dazu bei, dass man länger im Arbeits- 
markt verbleiben kann, der längere Verbleib im Arbeitsmarkt zur Investition in Weiterbildung.

ABBILDUNG 8 HIER

Zwar bestehen erhebliche Probleme bei der Definition und statistischen Erfassung von Weiterbildung auf nationaler Ebene und im internationalen Vergleich. Man kann aber gleichwohl festhalten, dass unabhängig von der Quelle das Grundmuster der Interpretation stabil bleibt: An Weiterbildung sind in Deutschland Älteren und gering Qualifizierte kaum beteiligt. Im Wesentlichen gibt es eine Konzentration auf die Personen, die schon vergleichsweise viel Humankapital in den Arbeitsmarkt mitbringen und bei denen Weiterbildung für die Betriebe unmittelbar lohnend erscheint (vgl. hierzu auch Büchel/Pannenberg 2004).

Dies begünstigt Personen, die von der in Deutschland sehr stark ausgebauten beruflichen oder akademischen Erstausbildung profitieren. Hier besteht eine Schnittstelle zur schulischen, beruflichen und akademischen Erstausbildung. Ungleichheiten aus der Erstausbildung setzen sich letztlich im weiteren Verlauf des Erwerbslebens fort. Andere Gruppen, die wesentlich stärker Gefahr laufen, arbeitslos zu werden, haben in geringem Maße Zugang zu Weiterbildung, außer in dem Fall, dass sie bereits arbeitslos sind und an einer öffentlich geförderten Qualifizierungsmaßnahme teilnehmen können, welche dann aus Mitteln der aktiven Arbeitsmarktpolitik finanziert wird. In Deutschland gibt es zwar ein gut ausgebautes System der Weiterbildung im Bereich der aktiven Arbeitsmarktpolitik. Dagegen existieren relativ wenige Regelungen, die dazu beitragen, dass Personen an Weiterbildung teilhaben, die eben nicht von der arbeitgeberseitigen Fortbildung profi- 
tieren. Diese wiederum konzentriert sich im Wesentlichen auf die höher Qualifizierten.

Neben den mittelbaren Effekten einer über Jahrzehnte hinweg praktizierten Frühverrentungsstrategie spielen aber auch institutionelle Faktoren im Bildungs- und Ausbildungssystem selbst eine wichtige Rolle bei der Erklärung der zu beobachtenden Situation im Feld der Weiterbildung. So besteht in eine strikte institutionelle Trennung zwischen den verschiedenen Teilsystemen der Aus- und Weiterbildung im Hinblick auf Steuerung und Finanzierung. Dies gilt für die Trennung zwischen Aus- und Weiterbildung ebenso wie für die Spaltung zwischen Sozialund Arbeitsmarktpolitik und Bildungspolitik: Während die schulische und akademische Ausbildung von den Ländern verantwortet wird, ist die Arbeitsmarktpolitik eine Bundesangelegenheit, die berufliche Ausbildung aber eine gemischte Verantwortlichkeit von Arbeitgebern, Gewerkschaften und Staat, während die Weiterbildung überwiegend von den Arbeitgebern getragen wird.

Das erklärt zum Teil die Schwierigkeiten, eine institutionelle Lösung im Bereich der Weiterbildung zu finden. Die Diskussion über lebenslanges Lernen als Stichwort und Notwendigkeit sowie als Leitprinzip für die Reform von Arbeitsmarkt-, Bildungs- und Tarifpolitik wird bereits länger geführt, dagegen wurde aber in diesem Bereich nur wenig Konkretes unternommen. Gegenüber der erneuten Ausweitung von Transferansprüchen und verschiedenen Formen der öffentlich geförderten Beschäftigung wird die Weiterbildung strukturell vernachlässigt. Dies zeigt sich erneut in der jüngsten Diskussion um die Verlängerung des Arbeitslosengeldes I und die Ausweitung öffentlich geförderter Beschäftigung, die aus dem Haushalt der Arbeitsmarktpolitik finanziert werden, während die Ausgabenbereitschaft im Bereich einer präventiven Weiterbildung auch für Personen, die nicht in den Genuss arbeitgeberfinanzierter Weiterqualifizierung kommen, nur gering ist. So 
steht etwa das 2007 vom Bundesbildungsministerium vorgelegte Modell des „Weiterbildungssparens" unter der Maxime der Haushaltsneutralität (Dohmen/de Hesselle/Himpele 2007, Rürup/Kohlmeier 2007), für das verlängerte Arbeitslosengeld I sind ebenso wie für die erweiterten Instrumente der aktiven Arbeitsmarktpolitik Mehrausgaben von mehreren Milliarden Euro jährlich zu erwarten. Dies entspricht einem generell zu beobachtenden Muster, dass Mehrausgaben für die kurzfristig verfügbare Kompensation von Arbeitslosigkeit durch aktive und passive Arbeitsmarktpolitik leichter zu realisieren sind als mittel- und langfristig wirksame Investitionen in die Aus- und Weiterbildung. Dies ist nicht nur eine Frage der politischen Präferenzen, sondern auch der institutionellen Routinen und der Finanzierungsform. Die Mobilisierung von Steuermitteln für eine präventive Strategie über Bildung ist schwieriger als die Verwendung von Beitragsmitteln für die Kompensation durch aktive und passive Arbeitsmarktpolitik (Nikolai 2007).

\section{Reformüberlegungen - Von der Kompensation zur Prävention}

5.1 Ausgangslage: Primär kompensierende Politik, strukturelle Vernachlässigung der Weiterbildung

Zwar hat sich die Beschäftigungssituation älterer Erwerbspersonen in Deutschland in den letzten Jahren deutlich verbessert, wobei diese Entwicklung primär den Veränderungen im Bereich der Optionen zum vorzeitigen Ausstieg aus dem Arbeitsmarkt, den Vorboten des demographischen Wandels und schließlich auch dem gegenwärtig günstigen konjunkturellen Umfeld zuzuschreiben ist. Das Erwerbsleben wird damit im Durchschnitt wieder länger, nachdem über Jahrzehnte 
eine Verkürzung aus arbeitsmarktpolitischen Gründen präferiert worden war. Dabei dominiert aber die Weiterbeschäftigung in bestehenden Arbeitsverhältnissen, während die Wiederbeschäftigung nach einmal eingetretener Arbeitslosigkeit noch immer im späteren Erwerbsleben schwieriger ist als in jüngeren Jahren. Hierin spiegeln sich noch immer gegebene Sonderregelungen für Ältere in den Transferleistungen und der Aktivierung ebenso wider wie das Erbe einer strukturellen Vernachlässigung der beruflichen Weiterbildung, insbesondere im Bereich der Entwicklung und Anpassung von übertragbarem, also nicht betriebsspezifischem Wissen.

Weiterbildung ist in Deutschland sehr stark auf höher qualifizierte und jüngere Arbeitskräfte konzentriert, während die Personen, die aufgrund geringer formaler Qualifikation am stärksten Gefahr laufen, im Strukturwandel nicht Schritt halten zu können und arbeitslos zu werden, weder aus eigener Initiative noch durch arbeitgeberfinanzierte Weiterbildung in ihre Beschäftigungsfähigkeit investieren. Arbeitsmarktpolitische Maßnahmen setzen in Deutschland primär bei einmal eingetretener Arbeitslosigkeit ein und zeigen dann eine insgesamt wenig beeindruckende Wirksamkeit im Hinblick auf die Wiedereingliederung in Beschäftigung. Maßnahmen speziell für Ältere kommen also prinzipiell zu spät und setzen darüber hinaus im Kern an der Kompensation von eingetretenen Qualifikationsdefiziten an, etwa durch Lohnkosten- oder Entgeltzuschüsse oder den Verweis auf überwiegend öffentlich finanzierte Beschäftigungsprogramme ohne Eingliederungsziel. Gemeinsam mit den noch bestehenden Alternativen, in den Bezug von Transferleistungen zu wechseln, steht dieser Ausgaben- und Programmschwerpunkt einer präventiven Anpassung beruflich nutzbarer Qualifikationen im gesamten Verlauf des Erwerbslebens („lebenslanges Lernen“) entgegen. 
Zwar wird für Arbeitgeber und Arbeitnehmer die Weiterbildung bei längerem

Erwerbsverlauf reizvoller und damit attraktiver, jedoch profitieren von arbeitgeberseitiger und arbeitnehmerseitiger Investition nur jüngere, höher qualifizierte Personen. Beruflich nutzbare Qualifikationen sollten jedoch soweit erworben und weiterentwickelt werden, dass möglichst alle Erwerbspersonen im weiteren Verlauf eines (längeren) Erwerbslebens ohne großen Subventionierungsbedarf in Gestalt von Eingliederungszuschüssen oder Kombilöhnen im Arbeitsmarkt mithalten können.

\subsection{Institutionalisierung von Weiterbildung}

Weiterbildung als frühzeitige Investition, vor drohender oder eingetretener Arbeitslosigkeit, kann die Beschäftigungs- und Aufstiegsperspektive im Erwerbsverlauf positiv beeinflussen (OECD 2006). Wie lässt sich erreichen, dass die Beteiligung an Weiterbildung im Lebensverlauf stabilisiert wird und auch für Erwerbspersonen mit weniger hoher Qualifikation zunimmt? Dies bedarf eines einfachen und leicht handhabbaren Modells der Kofinanzierung, das mit entsprechenden Strukturen im Markt für Weiterbildung verbunden wird.

Derzeit wird die Weiterbildung der Erwerbstätigen primär von den Unternehmen getragen; daneben besteht die Möglichkeit, Aufwendungen für Weiterbildung steuerlich geltend zumachen, und zwar sowohl für Arbeitgeber als auch für Arbeitnehmer im Rahmen der Berücksichtigung als Betriebsausgaben bzw. Werbungskosten. Konzeptionell wurde zuletzt das „Weiterbildungssparen“ als Verbindung von privaten Ressourcen aus Ersparnissen, der steuerlich geförderten Vermögensbildung oder zinsgünstigen Darlehen mit öffentlicher Förderung über 
Steuervergünstigungen oder einer direkten Prämie diskutiert (Dohmen/de Hesselle/Himpele 2007, Rürup/Kohlmeier 2007). Hier besteht das Problem jedoch darin, dass die Logik des Weiterbildungssparens und die vergleichsweise kleinteilige Struktur dieses Modells nicht dazu geeignet sein dürfte, die Zielgruppe entsprechend zu erreichen und zu motivieren, in Weiterbildung zu investieren. Eine sinnvolle und leicht handhabbare Lösung könnte vielmehr in einem Weiterbildungsgutschein liegen, der eine öffentliche Teilfinanzierung von Weiterbildungsaktivitäten vornimmt. Wichtig ist dabei die regelmäßige Weiterqualifikation. Die öffentlichen Finanzierungsanteile können dabei auf Personen mit geringer Qualifikation bzw. Einkommen konzentriert werden, indem der Förderanteil bei diesen besonders hoch ausfällt oder sie häufiger in den Genuss von Gutscheinen kommen.

Was die Finanzierungsseite angeht, so sind Individuen, Arbeitgeber und öffentliche Hand gleichermaßen gefragt. Der einzelne Arbeitnehmer hat einen Nutzen aus Weiterbildungsanstrengungen, da seine Chancen auf beruflichen Aufstieg und stabile Beschäftigung zunehmen. Grundprinzip sollte es deshalb sein, dass die individuellen Weiterbildungsanstrengungen letztlich so selbstverständlich werden wie private Altersvorsorge, und zwar auch für Personen, die derzeit noch nicht in Weiterbildung investieren. Dies ist eine durchaus treffende Analogie: nicht nur bei der Altervorsorge nimmt die Rolle der Eigenverantwortung zu, sondern auch bei der Sicherung der Teilhabe am Arbeitsmarkt, da es keineswegs ausreicht, sich auf öffentlich geförderte oder vom Arbeitgeber bereitgestellte Weiterbildungsmaßnahmen zu verlassen, um beschäftigungsfähig gehalten zu werden.

Arbeitgeber finanzieren primär betriebsspezifische Qualifikationen, oft in kurzfristiger Perspektive, profitieren jedoch auch von übertragbarem Humankapital, welches sich ohnehin nicht einfach abgrenzen lässt. Im Zusammenhang mit dem Gutschein für Weiterbildung wäre es denkbar, dass sich die Beteiligung der Arbeitge- 
ber entweder auf eine (steuerlich begünstigte) Zusatzfinanzierung oder die Freistellung innerhalb der Arbeitszeit bezieht.

Eine Mitfinanzierung seitens des Staates ist gerechtfertigt, da Weiterbildung dazu beiträgt, später anfallende Ausgaben für Transferleistungen und Maßnahmen der aktiven Arbeitsmarktpolitik zu vermindern. Um erhebliche Mitnahmeeffekte zu vermeiden, ist eine öffentliche Beteiligung dabei auf jene Personen zu konzentrieren, die ohne Förderung (und Beratung) nicht in Weiterbildung investieren würden. Dies spricht für eine nach Einkommen oder Qualifikation degressive Ausgestaltung. Derzeit wird beruflich relevante Weiterbildung in erster Linie die steuerliche Absetzbarkeit gefördert, was höhere Einkommen begünstigt.

Neben dem Gutscheinmodell selbst sind als wichtige Rahmenbedingungen für ein erfolgreiches Funktionieren eine unabhängige und verpflichtende Beratung der Gutscheininhaber über Weiterbildungsangebote und ein hohes Maß an Transparenz im Weiterbildungsmarkt hinsichtlich der Einhaltung von Qualitätsstandards und der Bewertung erworbener Qualifikationen nach einem Punktesystem zentral, welches auch die Anerkennung und Übertragbarkeit von Weiterbildungsleistungen gewährleisten würde. Gutscheine sollten nur bei zertifizierten Anbietern eingelöst werden können.

\subsection{Weiterbildung, Arbeitslosenversicherung und Kündigungsschutz}

Ein Gutscheinmodell schöpft das Potenzial von systematischeren Anreizen, in Weiterbildung zu investieren, nicht vollständig aus. So kann der Weiterbildungsgutschein einfach nicht in Anspruch genommen werden, ohne dass dies unmittel-

bare Wirkungen auf die Absicherung im Fall der Arbeitslosigkeit hat. Auch die 
Verbesserung der Beschäftigungsfähigkeit wirkt sich möglicherweise nicht so kurzfristig aus, dass die Erträge von Weiterbildungsinvestitionen etwa durch beruflichen Aufstieg oder vermiedene bzw. verkürzte Arbeitslosigkeit klar hervortreten. Deshalb ist zu überlegen, ob Veränderungen im Bereich der Sicherungsmechanismen gegen Arbeitsmarktrisiken wie dem Kündigungsschutz und insbesondere der Arbeitslosenversicherung so verändert werden, dass klare Anreize zur Vermeidung bzw. Verkürzung von Phasen der Arbeitslosigkeit und zu vorausschauendem Verhalten sowie Weiterbildung gesetzt werden.

Ein Modell hierfür ist die Gestaltung der Arbeitslosenversicherung als eine Beschäftigungsversicherung mit dem Charakter eines Guthabenkontos (vgl. hierzu auch Boss/Brown/Snower 2007, Schmid 2002), wobei eine solidarische Komponente in der Risikoabsicherung erhalten bleiben muss. Regelmäßige Einzahlungen seitens Arbeitgeber und Arbeitnehmer wären dabei nicht nur für die Abdeckung von Arbeitslosigkeit zu verwenden, sondern auch für die Finanzierung von Weiterbildungsaktivitäten oder die Aufstockung von Phasen mit geringem Einkommen etwa aufgrund von Teilzeitarbeit oder Zeiten geringerer Entlohnung, ähnlich etwa dem "Zwischenverdienst" in der Schweiz. Wird dadurch Arbeitslosigkeit verkürzt oder vermieden, so wächst das Guthaben an und kann als zusätzliche private Altersvorsorge betrachtet werden.

Dieses Modell wäre ähnlich der österreichischen „Abfertigung neu“ auch als eine Variante zur Reform des Kündigungsschutzes geeignet. Vom Bestandsschutz, der überwiegend zu Vereinbarungen über Abfindungen führt, könnte damit ein Übergang zu einem generellen Abfindungsmodell vollzogen werden, bei dem während der Beschäftigung regelmäßige Einzahlungen des Arbeitgebers auf das Konto der Beschäftigungsversicherung stattfinden. Damit könnten dann Investitionen in die Beschäftigungsfähigkeit, insbesondere auch in übertragbare Qualifikationen finan- 
ziert werden, welche im Falle einer Beendigung des Arbeitsverhältnisses helfen, die Stellensuche zu erleichtern. In dem Sinne wären Leistungen des Arbeitgebers weniger als Entschädigung für Entlassungen denn als Vorbereitung auf innerbetriebliche Veränderungen oder eben neue Jobs zu verstehen. Ein primär auf den Bestandsschutz abzielender Kündigungsschutz stabilisiert zwar die Beschäftigung, vermindert aber die Chancen auf Wiedereinstiege und trägt zur Spaltung des Arbeitsmarktes bei. In dem Maße wie über ein Kontenmodell in der Arbeitslosenversicherung und intensivere Weiterbildung die Absicherung gegen Arbeitsmarktrisiken durch bessere Beschäftigungsfähigkeit gewährleistet wird, wird der Kündigungsschutz weniger notwendig sein.

Im Zuge der Reform könnten auch Entnahmen aus dem Konto für die Finanzierung professioneller Bemühungen um Wiedereingliederung genutzt werden. Hier sollten die Erfahrungen mit den Transfermaßnahmen in der aktiven Arbeitsmarktpolitik (IZA/DIW/Infas 2006) oder mit den österreichischen "Arbeitsstiftungen“ berücksichtigt werden (Winter-Ebmer 2006). Grundsätzlich sollten solche Instrumente nicht nur bei Massenentlassungen, sondern auch im Einzelfall nutzbar sein und nicht primär Arbeitslosigkeit abfedern, sondern eine rasche Wiedereingliederung erreichen. Erfahrungen liegen auch in Deutschland mit dem Vermittlungsgutschein oder der Einschaltung Dritter bei der Vermittlung vor. Eine frühzeitige Mobilisierung professioneller Dienstleister, auch von Zeitarbeit, privaten Arbeitsvermittlern und Outplacement-Beratern, könnte Phasen der Arbeitslosigkeit deutlich verkürzen. 


\section{Fazit}

\subsection{Generelle Maxime: Ein altersneutrales Arrangement auf dem Arbeitsmarkt}

Für die Zukunft ist angesichts des demographischen Wandels mit einer weiteren Verbesserung der Beschäftigungssituation älterer Arbeitskräfte zu rechnen. Einerseits stehen weniger jüngere Arbeitskräfte bereit, andererseits wirken die Veränderungen in den Transfersystemen auf das Verhalten von Arbeitgebern und Arbeitnehmern ein, wie dies derzeit zu beobachten ist. Diese beiden Faktoren sprechen dafür, dass sich der Aufwärtstrend bei der Beschäftigung Älterer fortsetzen wird und im Prinzip mit einer weiteren „Normalisierung" relativ zu der Beschäftigungssituation jüngerer Arbeitskräfte gerechnet werden kann. Ein längerer Verbleib im Arbeitsmarkt wird damit zunehmend selbstverständlich. Dies gilt umso mehr für die Zukunft, je klarer und eindeutiger die Anreize innerhalb der Transfersysteme ausgestaltet sind und langfristig stabil gehalten werden. Dieser über mehrere Jahre hinweg anhaltende Trend könnte jedoch gedämpft werden, wenn die Anreize zum Verbleib im Arbeitsmarkt erneut abgeschwächt werden.

Die „Normalisierung“ der Beschäftigungssituation älterer Arbeitskräfte und die Verlängerung des Erwerbslebens sprechen dafür, noch bestehende altersabhängige Differenzierungen bei Transferleistungen, Ausnahmen vom Grundsatz der Aktivierung, aktiven arbeitsmarktpolitischen Maßnahmen, aber auch bei der arbeitsrechtlichen Regulierung abzubauen und „altersneutrale“ Rahmenbedingungen zu schaffen. Die Sonderregelungen für Ältere wirken sich mittelbar negativ auf die Beschäftigung Älterer und auf Investitionen in die Beschäftigungsfähigkeit aus. Ein „altersneutraler“ Arbeitsmarkt basiert auf einer präventiven, auf den Erhalt 
der Beschäftigungsfähigkeit im Erwerbsverlauf ausgerichteten Politik mit einer stärkeren Betonung von beruflicher Weiterbildung und Anpassungsfähigkeit, während ein ausgeprägter Bestandsschutz für Ältere, kompensierende Transferleistungen und öffentlich subventionierte Beschäftigung an Bedeutung verlieren werden. Umstiege und Wiedereinstiege auch in einem späteren Stadium des Erwerbslebens werden damit erleichtert. Dies wird umso eher gelingen, je rascher ein stabiles und in sich konsistentes Arrangement auf dem Arbeitsmarkt realisiert werden kann.

\subsection{Aktivierung unabhängig vom Alter}

Das Beschäftigungsniveau kann zum einen durch eine konsequente Aktivierung von Älteren mittels des Prinzips Fördern und Fordern weiter gesteigert werden. Hier sollten keine Rückschritte vollzogen, sondern an einer weiteren Intensivierung gearbeitet werden, so dass noch vorhandene Ausnahmen wegfallen. Dazu gehört unter anderem die definitive Abschaffung des § 428 SGB III sowie des § 65 Abs. 4 SGB II, die im Prinzip bedeuten, dass Personen innerhalb der Arbeitslosenversicherung bzw. Grundsicherung im Gegensatz zu jüngeren Arbeitsuchenden eine Transferleistung erhalten können, ohne dem Arbeitsmarkt zur Verfügung zu stehen. Aus Sicht des Arbeitsmarktes spricht auch wenig dafür, die Bezugsdauer des Arbeitslosengeldes I an das Lebensalter oder die Beitragszahlung zu koppeln. Dies ist zum einen unter dem Aspekt der Gerechtigkeit problematisch, da jüngere Erwerbspersonen wesentlich stärker den "Zumutungen“ des Arbeitsmarktes und

einer aktivierenden Arbeitsmarktpolitik ausgesetzt sind als Ältere, die zumindest zu einem großen Teil ohne Einschränkungen erwerbsfähig sind und deshalb auch 
dem Arbeitsmarkt zur Verfügung stehen sollten. Zum anderen entwerten solche Transferoptionen den Ertrag von Weiterbildungsanstrengungen und legen ein produktiv nutzbares Arbeitskräftepotenzial still. Fallen diese Regelungen weg, so hat dies nicht nur Auswirkungen auf die Arbeitssuche und damit die Chance auf Aufnahme einer Erwerbstätigkeit, sondern auch auf die Intensität der Betreuung durch die Bundesagentur für Arbeit bzw. Arbeitsgemeinschaften und Optionskommunen. Es besteht prinzipiell keine Notwendigkeit, nach dem Alter zu differenzieren und bestimmte Gruppen von der Verfügbarkeit für Arbeit auszunehmen. Der Beitrag der aktiven Arbeitsmarktpolitik wird eher gering ausfallen, die Wirkung veränderter Transferleistungen und Aktivierungsstrategien ist dagegen wesentlich stärker einzuschätzen. Aktive Arbeitsmarktpolitik sollte sich an Problemlagen orientieren, nicht am Alter als Merkmale der Förderfähigkeit bzw. bedürftigkeit, um eine pauschale Zuschreibung von Defiziten zu vermeiden. In dem Sinne ist es wenig hilfreich, pauschal Instrumente für Personen über 50 oder $55 \mathrm{zu}$ entwickeln oder auszubauen - wichtiger wäre es, die eigentlichen Problemlagen anzugehen, insbesondere Qualifikationsprobleme, die unabhängig vom Alter auftreten können.

\subsection{Institutionalisierung von Lebenslangem Lernen}

Weiterhin offen ist, wie Weiterbildung stärker institutionalisiert und zu einem gewissen Teil auch öffentlich gefördert werden kann. Vor allem bedarf es einer Lösung dafür, wie bei den geringer Qualifizierten für einen praktischen Zugang zur Weiterbildung gesorgt werden kann, um das Arbeitslosigkeitsrisiko bei dieser Personengruppe zu entschärfen. Ein Gutscheinmodell stellt eine einfache und prakti- 
kable Lösung dar. Es dürfte mittelfristig auch Sinn machen, Weiterbildung mit der Arbeitslosenversicherung und dem Kündigungsschutz in Verbindung zu bringen, möglicherweise im Sinne eines Kontenmodells, bei dem einerseits gewährleistet sein muss, dass es keinen Anreiz zu längerer Arbeitslosigkeit gibt, aber andererseits einen relativ starken Antrieb, in die Beschäftigungsfähigkeit zu investieren. Dies muss auf der einen Seite von den Erwerbspersonen selbst, auf der anderen Seite aber mittels einer Kofinanzierung seitens des Arbeitgebers und der öffentlichen Hand geschehen. Letztere würde mittelbar selbst davon profitieren, wenn weniger Transferleistungen und Aufwendungen für Maßnahmen der aktiven Arbeitsmarktpolitik für Arbeitslose anfallen würden.

6.4 Flexibilisierung des Arbeitsmarktes

Der Bestandsschutz für etablierte Arbeitsverhältnisse in Gestalt des ausgeprägten Kündigungsschutzes für Ältere stabilisiert in der gegenwärtigen Situation die Beschäftigung im höheren Lebensalter, da ein Personalabbau auf Kosten der staatlichen Sozialpolitik weniger leicht möglich ist als früher. Gleichzeitig trägt er aber mittelbar zu einer Spaltung des Arbeitsmarktes und zu einer Schmälerung der Wiedereinstiegschancen bei. Dies spricht auf mittlere Sicht für ein größeres Maß an Flexibilität im Arbeitsrecht und die Annäherung von befristeten und unbefristeten Arbeitsverträgen. Weniger strikter Bestandsschutz erhöht tendenziell die Attraktivität von Investitionen in die Beschäftigungsfähigkeit. Hier liegen insbesondere Beispiele aus den Ländern wie Großbritannien oder Dänemark vor, in denen es einen relativ flexiblen Arbeitsmarkt gibt und wo die Weiterbildungsbeteiligung auch bei Älteren vergleichsweise hoch ist. In dem Maße, wie also die Weiterbildungsin- 
tensität zunimmt, sollte auch der Kündigungsschutz gelockert und Abfindungszahlungen primär in Investitionen in beruflich nutzbare Qualifikationen umgeleitet werden.

\subsection{Konsistente und zeitlich abgestimmte Reformschritte}

Besonders wichtig ist die Konsistenz und langfristiger Stabilität der einzelnen Politikschritte, damit sich die Akteure auf dem Arbeitsmarkt, d.h. Arbeitnehmer und Arbeitgeber, an verlässlichen Rahmenbedingungen orientieren und sich daran anpassen können. Bei einer konsistenten Ausgestaltung der Rahmenbedingungen ist davon auszugehen, dass sich die Beschäftigungssituation der Älteren im Vergleich zu der der Jüngeren deutlich normalisieren dürfte.

Die gegenwärtige Diskussion in Deutschland führt jedoch zu widersprüchlichen Anreizen, die weder konsistent noch langfristig stabil sein werden. Grundprinzip sollte es aber sein, ein „,altersneutrales“ Regime auf dem Arbeitsmarkt sowohl bei

der aktiven Arbeitsmarktpolitik als auch bei den Transferleistungen und der Regulierung zu etablieren. Das schließt Strukturen aus, die mittelbar die Beschäftigung von Älteren hemmen, und zielt darauf ab, das gesamte Arbeitskräftepotenzial, das angesichts des demographischen Wandels mobilisiert werden sollte, dem Arbeitsmarkt auch tatsächlich zur Verfügung zu stellen. Die momentan sehr günstige Situation der älteren Arbeitskräfte auf dem deutschen Arbeitsmarkt spricht dafür, die Laufzeit der noch vorhandenen Sonderregelungen in den Transfersystemen rasch zu beenden. Die sozialen Folgewirkungen würden nicht so massiv ausfallen wie in einer ökonomisch wesentlich schwierigeren Situation. 
Auf mittlere Sicht setzt die Verlängerung des Erwerbslebens jedoch auch voraus, dass der Bereich der Weiterbildung institutionell neu gestaltet wird. Dies ist allein deswegen notwendig, weil die Betriebe zwangsläufig wesentlich weniger auf jüngere Nachwuchskräfte zurückgreifen können und gleichzeitig die Arbeitnehmer seltener vorzeitig aus dem Arbeitsmarkt ausscheiden werden. Schon diese Tatsache wird einen positiven Effekt auf die Weiterbildungsaktivitäten ausüben und neue Lösungen auf der betrieblichen und tarifvertraglichen Ebene hervorbringen. Das wird aber allein nicht genügen.

Je mehr im Verlauf des Erwerbslebens in Weiterbildung investiert wird, umso weniger werden Wieder- und Weiterbeschäftigungsprobleme bei älteren Arbeitskräften auftauchen und umso geringer wird die Notwendigkeit aktiver Arbeitsmarktpolitik ausfallen, um Älteren einerseits das Humankapital zu verschaffen, was benötigt wird, um wieder in Arbeit zu gelangen und andererseits das Einkommen zu ermöglichen, was sie für die Erhaltung ihres Lebensstandards brauchen. Die Subventionierung von Beschäftigung durch Eingliederungszuschüsse, Kombilöhne und öffentliche Arbeitsgelegenheiten wird um so weniger notwendig sein, je kürzer Erwerbsunterbrechungen sind und je mehr bereits früher in Weiterbildung und damit Beschäftigungsfähigkeit investiert wird. Dies spricht für eine öffentliche Unterstützung von Weiterbildungsinvestitionen.

Die Herausforderung für Politik ist besteht aus heutiger Sicht darin, Zurückhaltung bei der Schaffung neuer Ausgabenverpflichtungen im kompensierenden Bereich zu üben, also bei Sozialleistungen und subventionierter Beschäftigung, die zwar einem kurzfristigen „sozialpolitischen Reflex“ folgen, aber dem für die Zukunft zentralen Bereich der Aus- und Weiterbildung knappe öffentliche Mittel entziehen. Damit werden die Anreize für Weiterbildung und vorausschauendes Verhalten bei Arbeitgebern und Arbeitnehmern eher geschwächt. 
Wichtig ist hierbei das Timing bei der Umsetzung einer konsistenten Reformstrategie. Es ist aus politischer Sicht plausibel zu argumentieren, dass die von den Reformen derzeit am stärksten betroffenen Altersjahrgänge mehr Berechtigung für eine bevorzugte Behandlung im Bereich der Kompensation durch Transferleistungen und öffentliche Beschäftigung haben, da sie sich in den letzten Jahren nicht rechtzeitig anpassen konnten, sondern lange in einem Regime tätig waren, das auf einen frühzeitigen Renteneintritt ausgerichtet war. Die Veränderung dieser Anreize kann bei den heute älteren Kohorten zu Härtefällen führen. Diese Situation darf aber nicht fortgeschrieben werden. Kompensierende Sonderregelungen im Transfersystem oder bei der Arbeitsmarktpolitik sowie Ausnahmen vom Prinzip der Aktivierung sind, wenn überhaupt, nur auf befristeter Basis zu vertreten. Damit muss eine klare Perspektive der Umorientierung und der Umschichtung knapper öffentlicher Ressourcen verbunden sein, um möglichst rasch ein klares und konsistentes Zielsystem für jüngere Kohorten zu schaffen, die dann genügend Zeit haben werden, um sich an die veränderten Rahmenbedingungen des Arbeitsmarktes anpassen zu können, also an ein längeres Erwerbsleben, weniger kompensierende Sozialpolitik und stärkere Betonung der Notwendigkeit von „lebenslangem Lernen". 


\section{Literaturverzeichnis}

Ammermüller, Andreas/Boockmann, Bernhard/Maier, Michael/Zwick, Thomas (2006): Eingliederungszuschüsse und Entgeltsicherung für Ältere. In: DIW Vierteljahrsheft 3/2006, 49-66.

Büchel, Felix/Pannenberg, Markus (2004): Berufliche Weiterbildung in West- und Ostdeutschland. In: Zeitschrift für Arbeitsmarktforschung 37 (2), 73-126.

Bellmann, Lutz/Kistler, Ernst/Wahse, Jürgen (2007): Demographischer Wandel: Betriebe müssen sich auf alternde Belegschaften einstellen. IAB Kurzbericht 21/2007. Nürnberg: IAB.

Boss, Alfred/Brown, Alessio J.G./Snower, Dennis J. (2007): Beschäftigungskonten für Deutschland. Kieler Arbeitspapier 1325. Kiel: Institut für Weltwirtschaft.

Brenke, Karl (2007): Die Bedeutung der Älteren auf dem Arbeitsmarkt nimmt deutlich zu. DIW Wochenbericht 74 (21), 337-345.

Brussig, Martin/Wojtkowski, Sascha (2007): Rückläufige Zugänge in Altersrenten aus sozialversicherungspflichtiger Beschäftigung - steigende Zugänge aus Arbeitslosigkeit. Altersübergangsreport 2007-02.

Bundesagentur für Arbeit (2007): Situation von Älteren auf dem Arbeitsmarkt. Nürnberg: Bundesagentur für Arbeit.

Büttner, Renate (2005): Zunehmende Erwerbsbeteiligung von Älteren. Altersübergangsreport 2005-04.

Dohmen, Dieter/de Hesselle, Vera/Himpele, Klemens (2007): Analyse möglicher Modelle und Entwicklung eines konkreten Konzepts zum Bildungssparen. Bonn: BMBF.

Eichhorst, Werner (2006): Beschäftigung Älterer in Deutschland: Der unvollständige Paradigmenwechsel. In: Zeitschrift für Sozialreform 52 (1), 101-123.

European Commission (2007): Employment in Europe 2007. Brüssel: European Commission.

Hirschenauer, Franziska (2007): Arbeiten bis 65 - längst noch nicht die Regel. IAB Kurzbericht 25/2007. Nürnberg: IAB.

IZA/DIW/Infas (2006): Evaluation der Maßnahmen zur Umsetzung der Vorschläge der Hartz-Kommission Modul 1d: Förderung beruflicher Weiterbildung und Transferleistungen. Juni 2006. Bonn: IZA. 
Lott, Margit/Spitznagel, Eugen (2007): Wenig Betrieb auf neuen Wegen der beruflichen Weiterbildung. IAB Kurzbericht 23/2007. Nürnberg: IAB.

Nikolai, Rita (2007): Sozialpolitik auf Kosten der Bildung? Verteilungskonkurrenz in Zeiten knapper Kassen. In: Zeitschrift für Sozialreform 53 (1), 7-30.

OECD (2005): Promoting Adult Learning. Paris: OECD.

OECD (2006): Ageing and Employment Policies - Live Longer, Work Longer. Paris: OECD.

Rürup, Bert/Kohlmeier, Anabell (2007): Wirtschaftliche und sozialpolitische Bedeutung des Weiterbildungssparens. Bonn: BMBF.

Schmid, Günther (2002): Wege in eine neue Vollbeschäftigung. Frankfurt am Main: Campus.

Steiner, Viktor (1997): Extended benefit entitlement periods and the duration of unemployment in West Germany. ZEW Discussion Paper 97/14. Mannheim.

Winter-Ebmer, Rudolf (2006): Coping with a structural crisis: evaluating an innovative redundancy-retraining project. In: International Journal of Manpower 27 (8), $700-721$.

ZEW/IAB/IAT (2006): Evaluation der Maßnahmen zur Umsetzung der Vorschläge der Hartz-Kommission: Arbeitspaket 1: Wirksamkeit der Instrumente. Modul 1d: Eingliederungszuschüsse und Entgeltsicherung. Bericht 2006. 


\section{Anhang}

Abbildung 1: Beschäftigungsquoten älterer Arbeitskräfte, 55-64 Jahre, 2000 und 2006

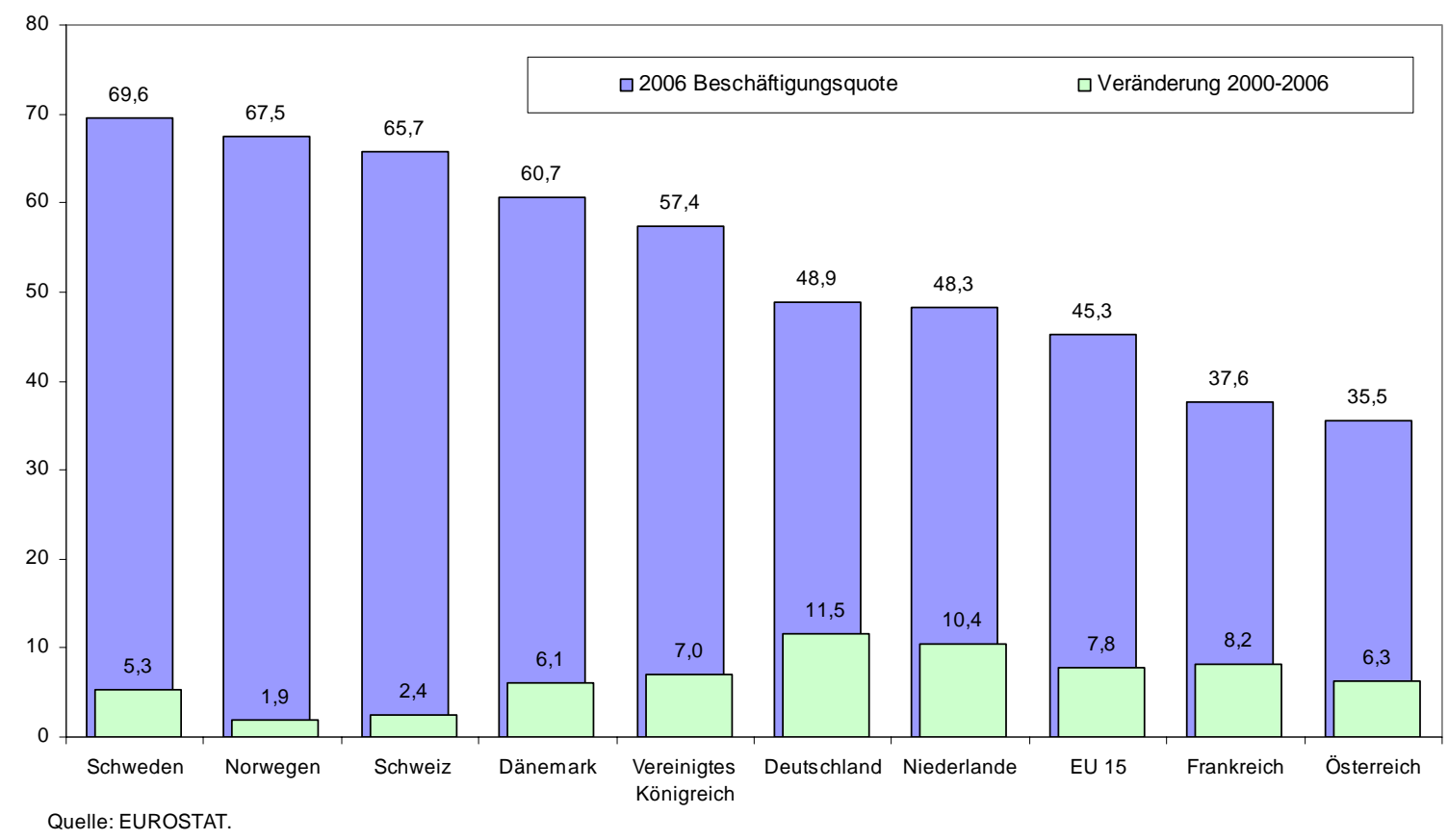


Abbildung 2: Differenz der Beschäftigungsquoten 15-64 Jahre / 55-64 Jahre in Prozentpunkten, 2000 und 2006

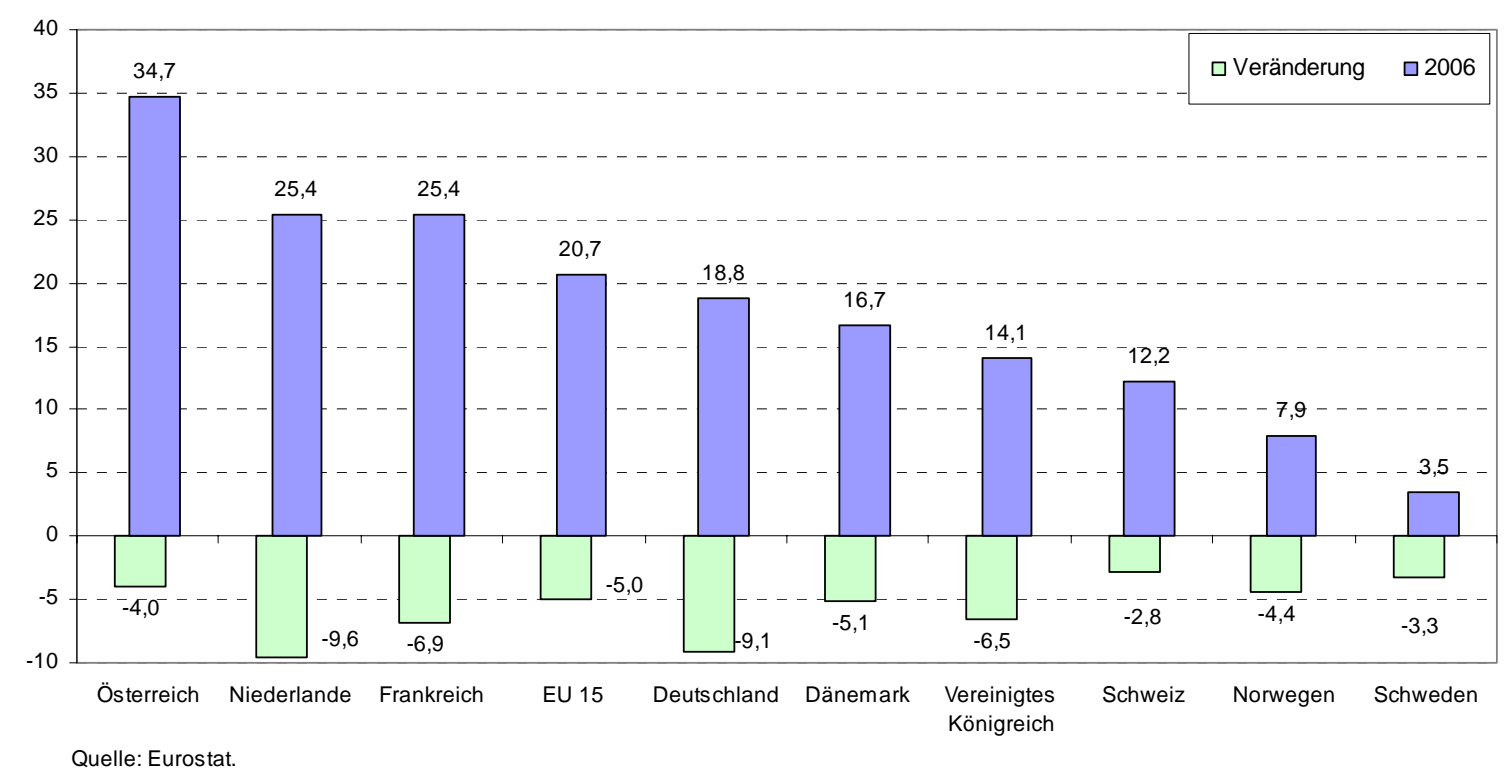

Quelle: Eurostat 
Abbildung 3: Sozialversicherungspflichtige Beschäftigung Älterer, März 2000 = 100

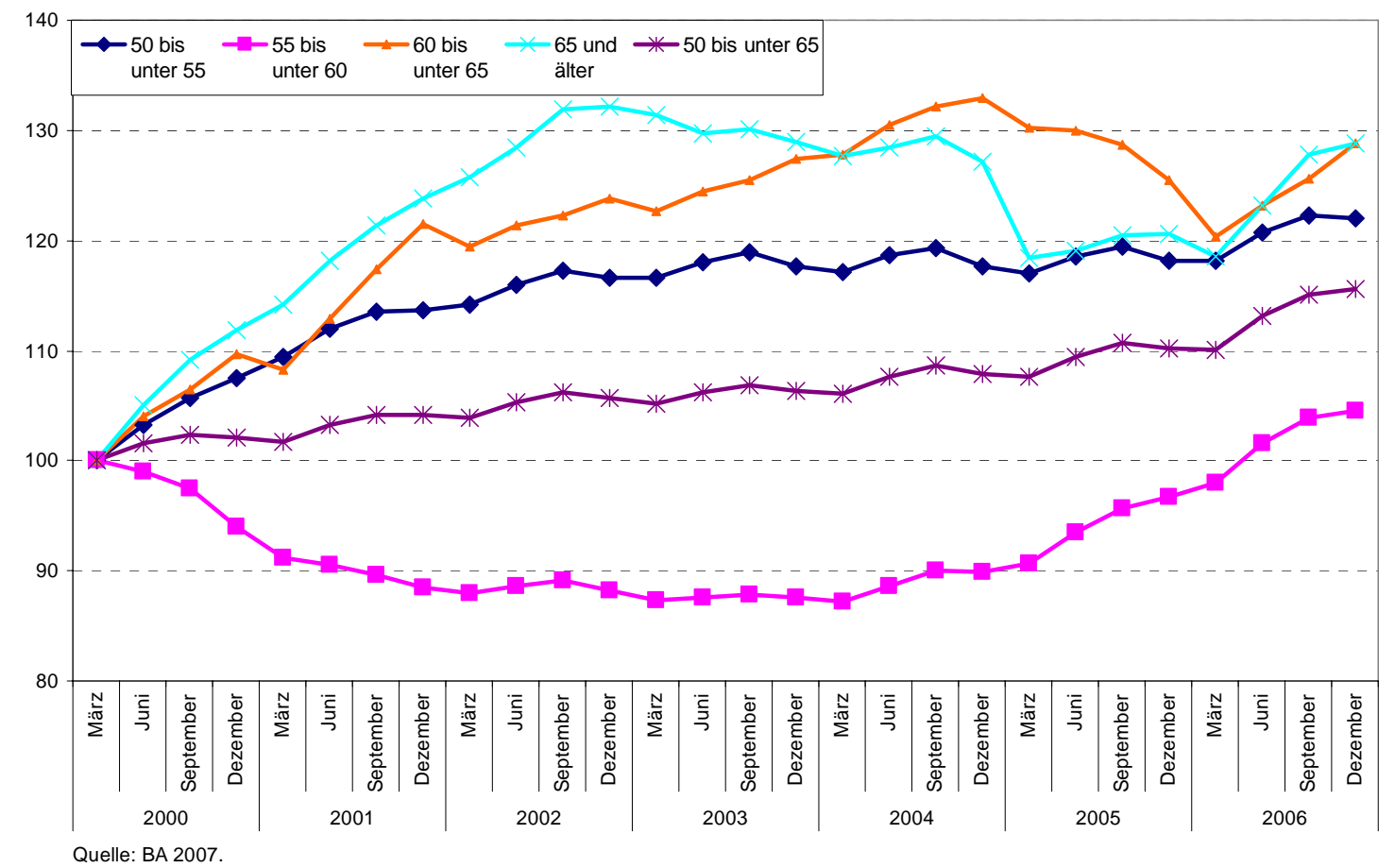


Abbildung 4: Faktisches Erwerbsaustrittsalter in Jahren, 2001 und 2006

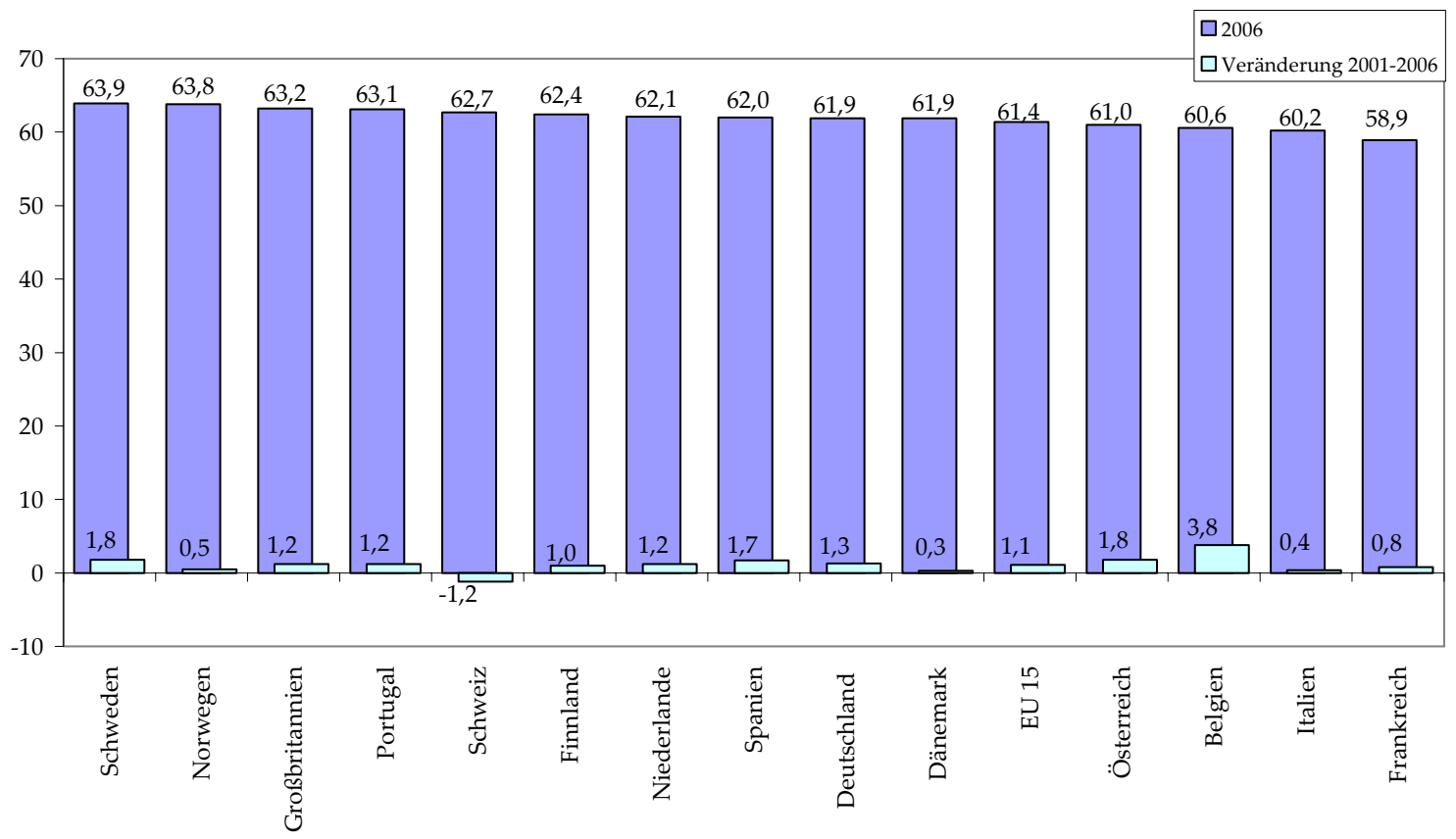

Quelle: Eurostat.

Portugal und Belgien: 2001 und 2005. 
Abbildung 5: Anteil der Langzeitarbeitslosen an allen Arbeitslosen in der Altersgruppe in \%, 2006

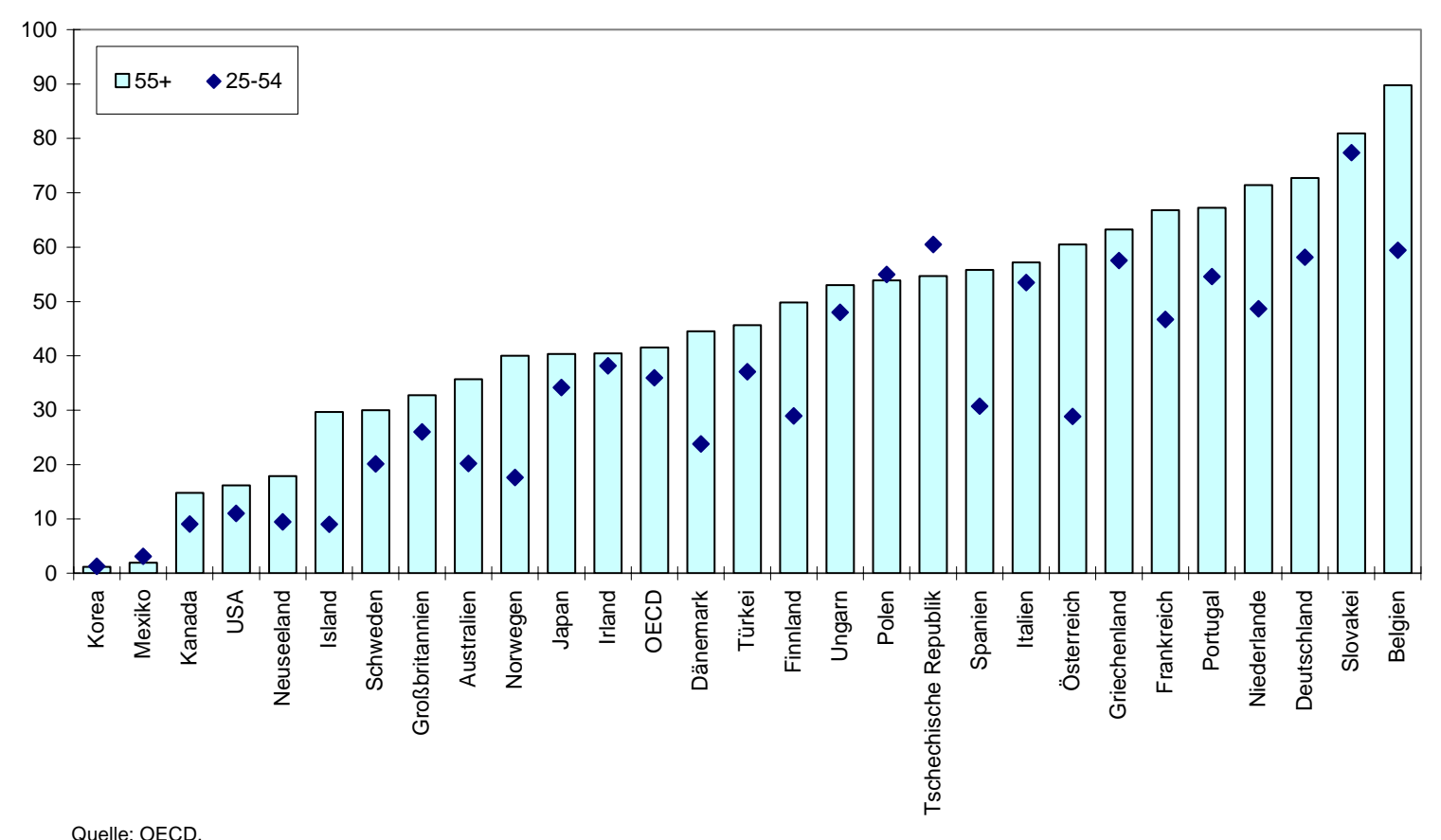


Abbildung 6: Einstellungswahrscheinlichkeit: Anteil der weniger als ein Jahr beim selben Arbeitgeber Beschäftigten in \% der Altersgruppe, 2006

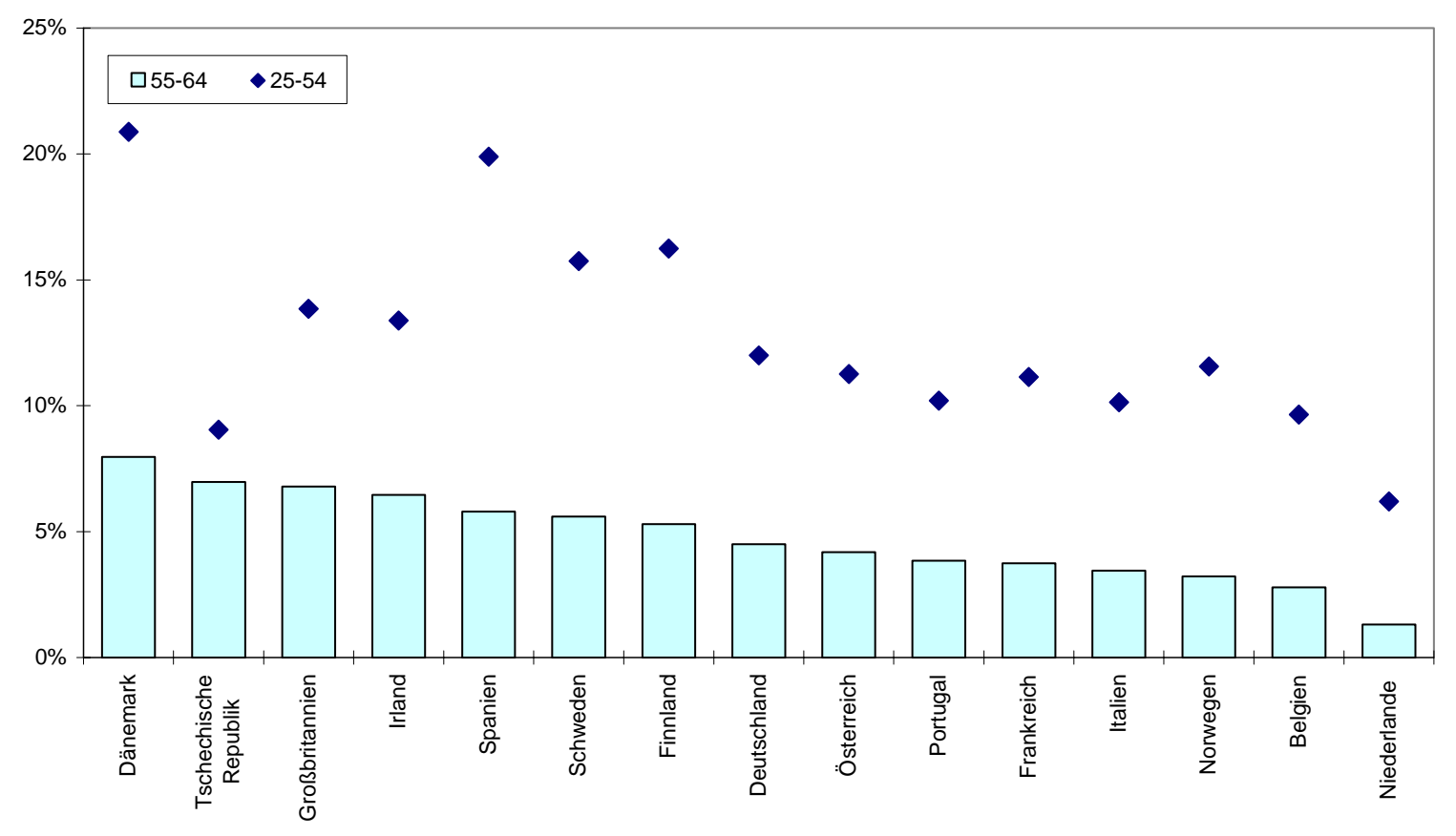

Quelle: OECD. 
Abbildung 7: Beschäftigungsquote von Älteren und Jüngeren, 2006

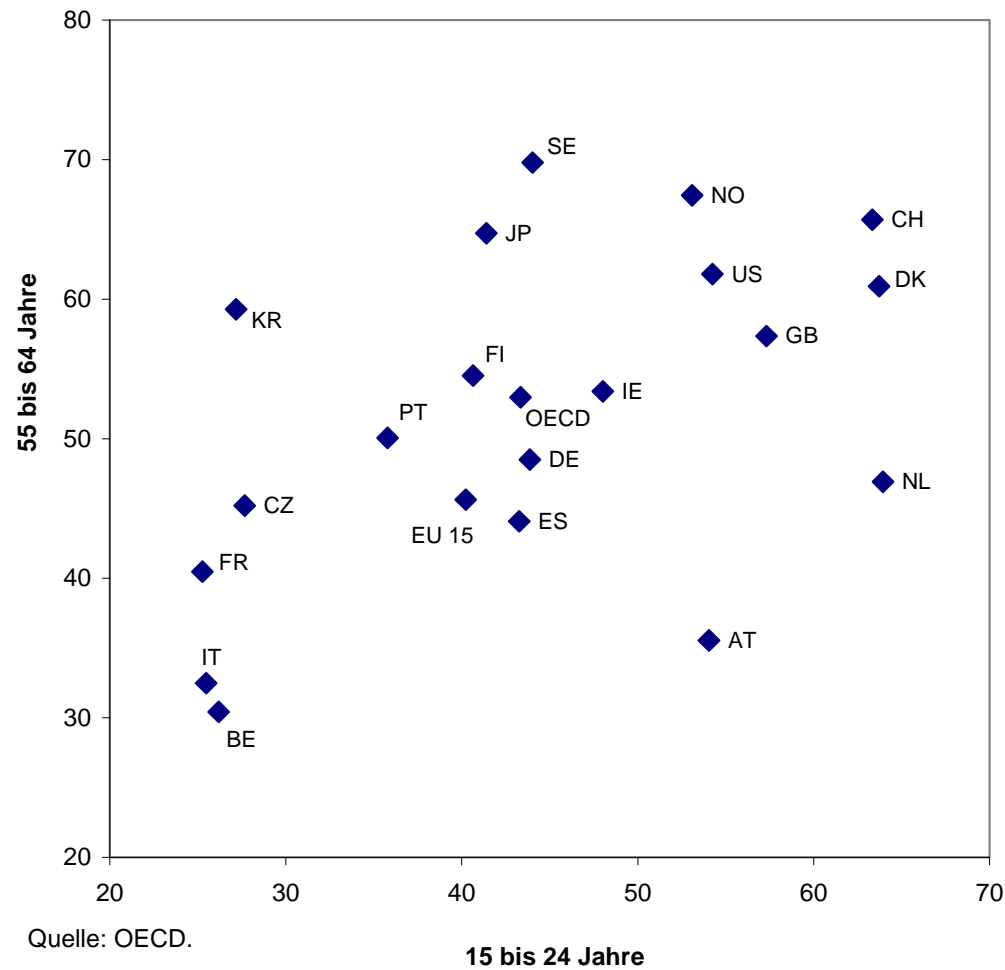


Abbildung 8: Verbleib auf dem Arbeitsmarkt und Teilnahme an Weiterbildung, 2005

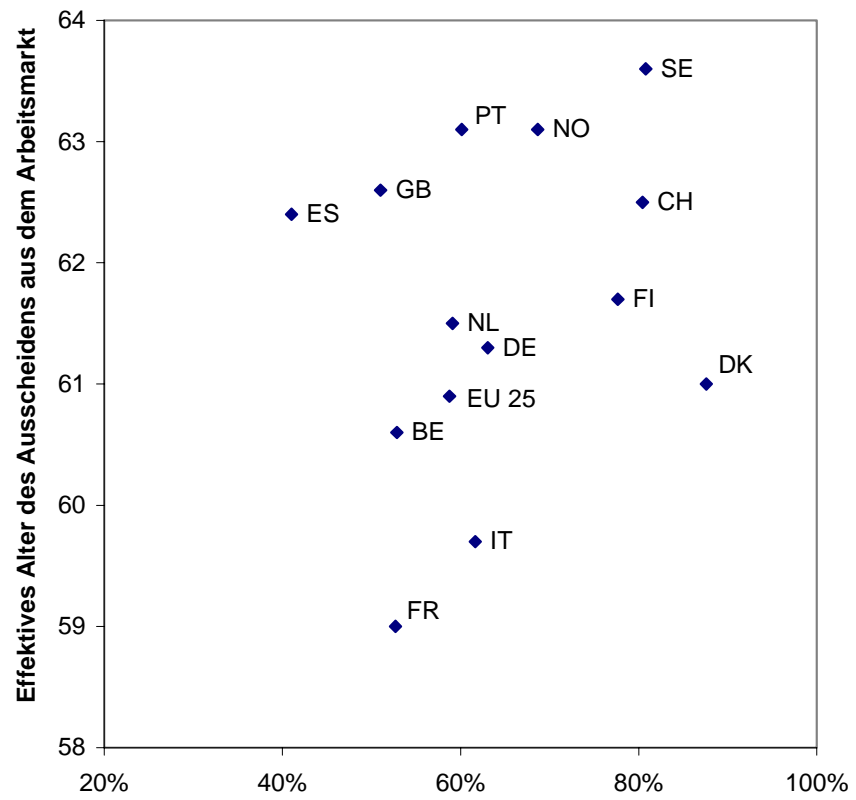

Teilnahmequote der Älteren an Weiterbildung in \% der Teilnahmequote der Jüngeren

Quelle: Eurostat. 
Tabelle 1: Erwerbs- und Erwerbstätigenquoten nach Bildungsabschluss sowie Qualifikationsstruktur der Erwerbspersonen und Erwerbstätigen im Alter von 50 bis 64 Jahren, in \%

\begin{tabular}{|c|c|c|c|c|c|}
\hline & 1995 & 1998 & 2000 & 2002 & 2004 \\
\hline \multicolumn{6}{|l|}{ Erwerbspersonen } \\
\hline \multicolumn{6}{|l|}{ Erwerbsquote } \\
\hline Ohne Abschluss & 38,8 & 39,4 & 39,4 & 41,5 & 44,8 \\
\hline Lehre, Fachschule & 57,9 & 56,4 & 55,4 & 57,3 & 59,9 \\
\hline Meister, Technikerschule & 72,4 & 70,1 & 69,0 & 70,1 & 71,5 \\
\hline Hochschule, Fachschule & 79,6 & 78,4 & 78,0 & 78,7 & 80,0 \\
\hline Insgesamt & 55,8 & 55,6 & 55,5 & 57,7 & 60,7 \\
\hline \multicolumn{6}{|l|}{ Qualifikationsstruktur } \\
\hline Ohne Abschluss & 20,0 & 18,2 & 16,6 & 15,1 & 14,5 \\
\hline Lehre, Fachschule & 55,9 & 56,0 & 56,8 & 58,5 & 57,7 \\
\hline Meister, Technikerschule & 9,0 & 9,4 & 9,8 & 9,7 & 9,5 \\
\hline Hochschule, Fachschule & 15,1 & 16,3 & 16,8 & 16,7 & 18,4 \\
\hline Insgesamt & 100,0 & 100,0 & 100,0 & 100,0 & 100,0 \\
\hline \multicolumn{6}{|l|}{ Erwerbstätige } \\
\hline \multicolumn{6}{|l|}{ Erwerbstätigenquote } \\
\hline Ohne Abschluss & 32,5 & 32,3 & 33,4 & 35,2 & 36,5 \\
\hline Lehre, Fachschule & 50,7 & 48,4 & 46,8 & 50,6 & 51,7 \\
\hline Meister, Technikerschule & 67,3 & 64,4 & 64,9 & 66,3 & 66,2 \\
\hline Hochschule, Fachschule & 75,1 & 73,3 & 73,8 & 74,9 & 75,2 \\
\hline Insgesamt & 49,2 & 48,3 & 49,4 & 51,6 & 53,1 \\
\hline \multicolumn{6}{|l|}{ Qualifikationsstruktur } \\
\hline Ohne Abschluss & 19,0 & 17,2 & 15,8 & 14,3 & 13,5 \\
\hline Lehre, Fachschule & 55,4 & 55,3 & 56,0 & 57,7 & 56,8 \\
\hline Meister, Technikerschule & 9,5 & 10,0 & 10,3 & 10,2 & 10,0 \\
\hline Hochschule, Fachschule & 16,2 & 17,5 & 17,9 & 17,8 & 19,7 \\
\hline Insgesamt & 100,0 & 100,0 & 100,0 & 100,0 & 100,0 \\
\hline
\end{tabular}


Tabelle 2: Teilnahme an Weiterbildung im Referenzzeitraum von vier Wochen, 25-64 Jährige

\begin{tabular}{|c|c|c|c|c|c|c|}
\hline & 2000 & 2001 & 2002 & 2003 & 2004 & 2005 \\
\hline Schweden & 21,6 & 17,5 & 18,4 & 31,8 & 32,1 & 32,1 \\
\hline Großbritannien & 20,5 & 20,9 & 21,3 & 26,8 & 29,4 & 27,5 \\
\hline Dänemark & 19,4 & 18,4 & 18,0 & 24,2 & 25,6 & 27,4 \\
\hline Schweiz & 34,7 & 36,0 & 34,4 & 24,7 & 28,6 & 26,9 \\
\hline Finnland & 17,5 & 17,2 & 17,3 & 22,4 & 22,8 & 22,5 \\
\hline Norwegen & 13,3 & 14,2 & 13,3 & 17,1 & 17,4 & 17,8 \\
\hline Niederlande & 15,5 & 15,9 & 15,8 & 16,4 & 16,4 & 15,9 \\
\hline Österreich & 8,3 & 8,2 & 7,5 & 8,6 & 11,6 & 12,9 \\
\hline EU-15 & 8,0 & 8,0 & 8,1 & 9,8 & 10,7 & 11,2 \\
\hline Spanien & 4,1 & 4,4 & 4,4 & 4,7 & 4,7 & 10,5 \\
\hline Belgien & 6,2 & 6,4 & 6,0 & 7,0 & 8,6 & 8,3 \\
\hline Deutschland & 5,2 & 5,2 & 5,8 & 6,0 & 7,4 & 7,7 \\
\hline Irland & : & : & 5,5 & 5,9 & 6,1 & 7,4 \\
\hline Frankreich & 2,8 & 2,7 & 2,7 & 7,0 & 7,0 & 7,0 \\
\hline Italien & 4,8 & 4,5 & 4,4 & 4,5 & 6,3 & 5,8 \\
\hline Portugal & 3,4 & 3,3 & 2,9 & 3,2 & 4,3 & 4,1 \\
\hline
\end{tabular}

Quelle: Eurostat. 
Tabelle 3: Durchschnittliche Stundenzahl an Weiterbildung pro Jahr nach Alter und Arbeitsmarktstatus, 2003

\begin{tabular}{|l|c|c|c|c|c|c|c|c|}
\hline & \multicolumn{4}{|c}{ Altersgruppe } & \multicolumn{3}{c|}{ Arbeitsmarktstatus } \\
\hline & $25-34$ & $35-44$ & $45-54$ & $55-64$ & Erwerbstätig & Arbeitslos & Inaktiv & Total \\
\hline Dänemark & 236 & 309 & 248 & 141 & 745 & 94 & 95 & 934 \\
\hline Schweden & 142 & 176 & 167 & 137 & 580 & 29 & 12 & 622 \\
\hline Schweiz & 254 & 205 & 177 & 87 & 637 & 47 & 39 & 723 \\
\hline Finnland & 191 & 221 & 180 & 77 & 528 & 85 & 55 & 669 \\
\hline $\begin{array}{l}\text { Großbritan- } \\
\text { nien }\end{array}$ & 119 & 97 & 71 & 28 & 269 & 14 & 33 & 315 \\
\hline Deutschland & 159 & 123 & 91 & 26 & 263 & 92 & 44 & 398 \\
\hline Frankreich & 366 & 206 & 118 & 23 & 493 & 102 & 117 & 713 \\
\hline Irland & 72 & 64 & 44 & 22 & 181 & $\mathbf{n}$ & 11 & 203 \\
\hline Niederlande & 122 & 87 & 53 & 21 & 231 & 10 & 41 & 283 \\
\hline Österreich & 169 & 141 & 92 & 20 & 373 & 20 & 29 & 422 \\
\hline Belgien & 197 & 163 & 89 & 20 & 378 & 53 & 37 & 469 \\
\hline Portugal & 162 & 111 & 54 & 16 & 260 & $\mathrm{n}$ & 23 & 343 \\
\hline Spanien & 105 & 73 & 47 & 11 & 177 & 37 & 20 & 237 \\
\hline Italien & 29 & 26 & 20 & 6 & 73 & 3 & 4 & 82 \\
\hline
\end{tabular}

Quelle: OECD. 
Tabelle 4: Durchschnittliche Stundenzahl an Weiterbildung pro Jahr nach Alter und Bildungsniveau, 2003

\begin{tabular}{|c|c|c|c|c|c|}
\hline & \multirow{2}{*}{ Bildungsniveau } & \multicolumn{4}{|c|}{ Altersgruppe } \\
\hline & & $25-34$ & $35-44$ & $45-54$ & $55-64$ \\
\hline \multirow{3}{*}{ Dänemark } & Niedrig & 239 & 243 & 171 & 65 \\
\hline & Mittel & 205 & 284 & 199 & 147 \\
\hline & Hoch & 282 & 379 & 362 & 207 \\
\hline \multirow{3}{*}{ Frankreich } & Niedrig & 245 & 118 & 75 & 12 \\
\hline & Mittel & 324 & 227 & 123 & 18 \\
\hline & Hoch & 488 & 291 & 206 & 76 \\
\hline \multirow{3}{*}{$\begin{array}{l}\text { Deutsch- } \\
\text { land }\end{array}$} & Niedrig & 54 & 39 & 32 & 5 \\
\hline & Mittel & 162 & 120 & 87 & 22 \\
\hline & Hoch & 243 & 187 & 153 & 66 \\
\hline \multirow{3}{*}{$\begin{array}{l}\text { Nieder- } \\
\text { lande }\end{array}$} & Niedrig & 92 & 73 & 41 & 11 \\
\hline & Mittel & 131 & 87 & 55 & 34 \\
\hline & Hoch & 130 & 103 & 67 & 22 \\
\hline \multirow{3}{*}{ Schweden } & Niedrig & 106 & 73 & 107 & 64 \\
\hline & Mittel & 123 & 164 & 149 & 125 \\
\hline & Hoch & 183 & 249 & 244 & 241 \\
\hline \multirow{3}{*}{$\begin{array}{l}\text { Großbri- } \\
\text { tannien }\end{array}$} & Niedrig & 30 & 35 & 27 & 12 \\
\hline & Mittel & 101 & 93 & 67 & 35 \\
\hline & Hoch & 161 & 140 & 117 & 62 \\
\hline
\end{tabular}

Quelle: OECD. 